\title{
Effects of different exercise training modes on muscle strength and physical performance in older people with sarcopenia: a systematic review and meta-analysis
}

Linqian Lu ${ }^{1,2+}$, Lin Mao ${ }^{3 \dagger}$, Yuwei Feng ${ }^{3 \dagger}$, Barbara E. Ainsworth ${ }^{4}$, Yu Liu ${ }^{1}$ and Nan Chen ${ }^{1,2^{*}}$

\begin{abstract}
Objective: We conducted a systematic review and meta-analysis to clarify the effects of different exercise modes (resistance training $[R T]$, whole body vibration training $[\mathrm{WBVT}]$, and mixed training $[M T$, resistance training combined with other exercises such as balance, endurance and aerobic training]) on muscle strength (knee extension strength [KES]) and physical performance (Timed Up and Go [TUG], gait speed [GS] and the Chair Stand [CS]) in older people with sarcopenia.

Method: All studies published from January 2010 to March 2021 on the effects of exercise training in older people with sarcopenia were retrieved from 6 electronic databases: Pubmed, Cochrane Library, Embase, Web of Science, the China National Knowledge Infrastructure (CNKI), and Wanfang Database. Two researchers independently extracted and evaluated studies that met inclusion and exclusion criteria. Pooled analyses for pre- and post- outcome measurements were performed using Review Manager 5.4 with standardized mean differences (SMDs) and fixed-effect models.
\end{abstract}

Result: Twenty-six studies (25 randomized controlled trails [RCTs] and one non-randomized controlled trail) were included in this study with 1191 older people with sarcopenia (mean age 60.6 \pm 2.3 to $89.5 \pm 4.4$ ). Compared with a control group, RT and MT significantly improved KES (RT, SMD $=1.36,95 \%$ confidence intervals [95\% Cl]: 0.71 to 2.02, $p<0.0001, I^{2}=72 \% ; M T, S M D=0.62,95 \% \mathrm{Cl}: 0.29$ to $\left.0.95, p=0.0002, \mathrm{I}^{2}=56 \%\right)$ and $\mathrm{GS}$ (RT, SMD $=2.01,95 \% \mathrm{Cl}: 1.04$ to $2.97, p<0.0001, I^{2}=84 \% ; M T, S M D=0.69,95 \% \mathrm{Cl}: 0.29$ to $\left.1.09, p=0.008, I^{2}=81 \%\right)$. WBVT showed no changes in KES (SMD $=0.65,95 \% \mathrm{Cl}:-0.02$ to $\left.1.31, p=0.06, \mathrm{I}^{2}=80 \%\right)$ or $\mathrm{GS}\left(\mathrm{SMD}=0.12,95 \% \mathrm{Cl}:-0.15\right.$ to $\left.0.39, p=0.38, \mathrm{I}^{2}=0 \%\right)$. TUG times were significantly improved with all exercise training modes (SMD $=-0.66,95 \% \mathrm{Cl}:-0.94$ to -0.38 , $\left.p<0.00001, I^{2}=60 \%\right)$. There were no changes in CS times with any of the exercise training modes (SMD $=0.11,95 \%$ Cl: -0.36 to $\left.0.57, p=0.65, I^{2}=87 \%\right)$.

Conclusions: In older people with sarcopenia, KES and GS can be improved by RT and MT, but not by WBVT. All three training modes improved TUG times, but not improved CS times.

\footnotetext{
*Correspondence: chennanreh2020@126.com

${ }^{\dagger}$ Linqian Lu, Lin Mao and Yuwei Feng contributed equally to this work.

${ }^{2}$ Department of Rehabilitation, Xinhua Hospital Chongming Branch,

Shanghai 202150, China

Full list of author information is available at the end of the article
}

(c) The Author(s) 2021. Open Access This article is licensed under a Creative Commons Attribution 4.0 International License, which permits use, sharing, adaptation, distribution and reproduction in any medium or format, as long as you give appropriate credit to the original author(s) and the source, provide a link to the Creative Commons licence, and indicate if changes were made. The images or other third party material in this article are included in the article's Creative Commons licence, unless indicated otherwise in a credit line to the material. If material is not included in the article's Creative Commons licence and your intended use is not permitted by statutory regulation or exceeds the permitted use, you will need to obtain permission directly from the copyright holder. To view a copy of this licence, visit http://creativecommons.org/licenses/by/4.0/. The Creative Commons Public Domain Dedication waiver (http://creativeco mmons.org/publicdomain/zero/1.0/) applies to the data made available in this article, unless otherwise stated in a credit line to the data. 
Keywords: Sarcopenia, Resistance training, Whole body vibration training, Mixed training, Muscle strength, Physical performance

\section{Background}

Sarcopenia is an age-dependent geriatric syndrome characterized by skeletal muscle mass loss, muscle strength and/or declines in physical performance [1].

A meta-analysis in 2020 indicated that the prevalence of sarcopenia was $9-10 \%$ in community-dwelling individuals, $30-50 \%$ in nursing-home individuals and $23-24 \%$ in hospitalized individuals [2]. Sarcopenia is associated with several adverse outcomes, including falls and secondary fractures [3, 4], pulmonary insufficiency [5], sleep disorders [6], cognitive impairment [7], poor quality of health-life [8] and premature mortality [9], all of which bring significant medical and economic burdens. Sarcopenia increases the risk of hospitalization of older people $[10,11]$. Total hospitalization costs are higher by nearly $\$ 13,000$ in preoperative older people with sarcopenia compared to older people without sarcopenia [12]. In 2000, the costs for sarcopenia-related conditions in the United States was $\$ 18.5$ billion, which represented 1.5\% of the annual medical expenditures [13]. Therefore, the prevention and treatment for sarcopenia is important to maintain physical function and improve health outcomes for older people and to reduce medical expenditures associated with sarcopenia.

To date, there are no effective pharmacological interventions to the treatment of sarcopenia [14]. Non-pharmacological interventions are the most appropriate and effective intervention for sarcopenia [15]. As a nonpharmacological intervention, exercise has been demonstrated by randomized controlled trails [RCTs] and meta-analysis to produce significant physiological and health benefits and to prevent and/or delay the development of sarcopenia [16-18]. The American College of Sports Medicine and the World Health Organization recommends that older people maintain $150 \mathrm{~min}$ of moderate-intensity aerobic exercise per week or $75 \mathrm{~min}$ of high-intensity aerobic exercise per week, and perform resistance exercise 2-3 times per week to prevent chronic or debilitating conditions and/or treat disease $[19,20]$. Previous studies have demonstrated that exercise (e.g., resistance training [RT], whole body vibration training [WBVT], mixed training [MT, such as resistance training combined with balance and aerobic training]) have a positive effect on increasing muscle mass [21], muscle strength [22] and physical performance [23]) in older people with sarcopenia.

Evidence has been provided from systematic reviews, meta-analyses and RCTs to show the efficacy of different exercise training modes on muscle mass, muscle strength and physical performance in older people with sarcopenia. Two reviews showed that RT can improve muscle strength and physical performance $[24,25]$ and that MT can increase physical performance [26]. Two meta-analyses also showed that RT is effective in improving muscle strength and physical performance and that WBVT has a positive effect on physical performance $[27,28]$. To date, little is known about the similarities and differences of RT, WBVT, MT exercise training modes and the effects of the study designs and/or protocols on muscle strength and physical performance.

Various methodologic and design weaknesses have consistently been found in exercise studies that limit the dissemination of exercise findings among older people with sarcopenia. First, there is no consensus on sarcopenia diagnostic criteria that makes studies included in previous meta-analysis inconsistent for the diagnostic criteria of sarcopenia [29]. In addition, there are differences in measurement methods for muscle strength and physical performance measures $[30,31]$, and exercise protocols $[32,33]$ that can lead to high heterogeneity in the results. Second, few studies have investigated the efficacy of different exercise modes to improve health outcomes and fitness levels in older people with sarcopenia. Alternatively, studies have focused on improving health outcomes and fitness in healthy older people [34], older people with osteoarthritis [35] or osteoporosis [36] or heart failure [37]. The aim of this study is to investigate the efficacy of three exercise training modes (RT, WBVT and MT) on knee extension strength (KES) and physical performance tests (Timed Up and Go [TUG], gait speed [GS] and the Chair Stand [CS]) to provide additional evidence for the treatment and management of sarcopenia in older people.

\section{Methods \\ Eligibility criteria}

The studies were included if they met the criteria for subjects and study types as follows: (a) age $>60$ years; (b) diagnosed with sarcopenia; (c) without diseases or conditions of COPD, cancer, kidney disease, hypertension, hyperlipidemia, stroke, diabetes, obesity, osteoporosis and fracture; (d) the study has at least one exercise group and one control group, and the control group must receive a no-exercise intervention or a health education course; (e) the exercise group must contain 
at least one type of RT, WBVT and/or MT exercise modes; and (f) each included study is the latest report since 2010.

The studies were excluded if they failed to meet the inclusion criteria and/or: (a) not full-text; (b) not in English or Chinese; (c) not a randomized controlled trial (RCT); (d) subjects were not diagnosed with sarcopenia previously in the exercise group and control group; (e) the exercise group received exercise interventions combined with nutritional supplementation; and (f) the study presented no extractable data.

\section{Search strategy}

This systematic review and meta-analysis is registered on the International Prospective Register of Systematic Reviews site (PROSPERO) as CRD42021256110. We followed the Preferred Reporting Items for Systematic Review and Meta-Analysis (PRISMA) guidelines [38]. A systematic search was conducted with the following six electronic databases from January 2010 to March 2021: Pubmed, Cochrane Library, Embase, Web of Science, the China National Knowledge Infrastructure (CNKI), and Wanfang Database. The studies in English and Chinese were all included. The following Mesh terms and the synonyms were used: "Sarcopenia", "Muscular Atrophy", "Aging", "Aged", "Frailty", "Randomized Controlled Trials", "Blind Method", "Exercise", "Exercise Therapy", "Resistance Training", "Endurance Training", "Vibration". We also used the terms "NOT (COPD, Cancer, Kidney Diseases)" (Full search strategy see Supplement 1).

\section{Study selection and data extraction}

Two researchers independently screened the title and abstract of each retrieved study to exclude irrelevant studies. Repeated studies for the same exercise RCT were excluded as were reviews of animal studies. The fulltext of each remaining study was systematically evaluated according to the inclusion and exclusion criteria. The bibliographic information for author, publication year, the characteristics of subjects (sample size, gender, mean age, and appendicular skeletal muscle mass/height ${ }^{2}$ $\left[\mathrm{ASM} / \mathrm{ht}^{2}\right]$ ), details of the exercise interventions (duration, type, frequency, and intensity), and the outcome measurements for body composition, muscle strength, and physical performance were independently extracted by two researchers. If a study was a multiple-arm trial, only the data of relevant exercise groups were extracted. A summary of the study results was recorded in a standard table format developed for this study. If information was recorded differently by the two researchers, a third researcher discussed the difference until it was resolved.

\section{Quality assessment}

Two researchers independently assessed the methodological quality of the studies using the Physiotherapy Evidence Database (PEDro) scale [39]. The following 11 characteristics were used to assess the quality of studies: Eligibility criteria, Random allocation, Concealed allocation, Baseline similar, Blinding (Subjects), Blinding (Therapists), Blinding (Assessors), Measure for $>85 \%$, Intention-to-Treat Analysis, Group comparison and Point measures. Each characteristic was rated as 0 (not meeting the criteria) to 1 (meeting the criteria). A high total score indicated higher study quality. When differences in ratings occurred between two researchers, a third researcher discussed the problem until it was resolved.

Two researchers independently assessed the risk of bias using the Review Manager (RevMan 5.4; Cochrane, Lindon, UK). Among the myriad of biases $[40,41]$ the bias assessment included selection bias, performance bias, detection bias, attrition bias and reporting bias. Each study was assigned a bias category of low risk, unclear risk or high risk. Biases not evaluated in this study were listed as other potential biases and assigned a category of unclear risk. Differences in the identification of study biases between the two researchers was resolved by discussion with a third researcher. Following agreement of bias for each study, the percentage of bias categories was calculated.

We used the Grades of Recommendation, Assessment, Development and Evaluation (GRADE) assessment to classify the overall certainty of evidence across studies of the outcomes and absolutely reduce the risk by using GRADE profiler version 3.6 [42] . The evidence of the outcomes of studies will be divided into one of the four grades [43]: (a) High: We are very confident that the true effect lies close to that of the estimate of the effect and progressive study is unlikely to change this result; (b) Moderate: We are moderately confident in the effect estimate, and the true effect is likely to be close to the estimate of the effect, but there is a possibility that it is substantially different; (c) Low: We have limited confidence in the results. Further research is very likely to have an important impact on our confidence on the estimate of effect and is likely to change the estimate; (d) Very low: We have little confidence in the results. The results may differ greatly from the real values, and further research is likely to change the results.

Originally, the evidence quality of RCTs is generally "High", and the following five factors will reduce the evidence quality to "Moderate", "Low" and "Very low": (a) Risk of bias [44]: without concealed allocation, without assessor blinded or other limitations; (b) Inconsistency [45]: excessive heterogeneity and thus inconsistency 
of results); (c) Indirectness [46]: indirect populations, interventions, controls and outcomes; (d) Imprecision [47]: relatively small simple size, wide confidence intervals, fewer studies; (e) Publication bias [48]: graphically in funnel plots or many potentially studies have not been published.

\section{Statistical analyses}

Review Manager (RevMan 5.4; Cochrane, Lindon, UK) was used to analyze all data. The statistical heterogeneity for the outcome in included studies was assessed by the $\mathrm{I}^{2}$ statistic. The analytic model used was dependent on the presence of heterogeneity; the fixed-effects model used when $\mathrm{I}^{2}<50 \%$ and random-effects model was used when $\mathrm{I}^{2}>50 \%$. To perform the meta-analysis, data with continuous outcomes were analyzed by the changes in the means and standard deviations (SD) of the outcome measurement. Weighted mean differences (WMD) and $95 \%$ confidence intervals $(95 \% \mathrm{CI})$ were computed when the studies had the same measurement methods and units for the independent and dependent variables.
Standardized mean differences (SMDs) and 95\% CI were computed when the studies had different measurement methods and units for the independent and dependent variables. In this meta-analysis, no studies met the criteria required to present results as WMD and 95\% CI. For studies where the mean and SD could not be extracted completely, the author was contacted in attempt to obtain the data. If the author could not be contacted, the study was excluded from analyses. $P<0.05$ was considered as statistical significance.

\section{Results}

\section{Study selection}

Figure 1 shows the flowchart for study screening and selection process according to the PRISMA guidelines. A total of 5889 records were retrieved, with 5730 retrieved using the keywords (Mesh terms and the synonyms) from Pubmed $(n=876)$, Cochrane Library $(n=815)$, Embase $(n=2436)$, Web of Science $(n=1603)$, the China National Knowledge Infrastructure (CNKI) $(n=107)$ and Wanfang Database $(n=32)$. Twenty studies were identified

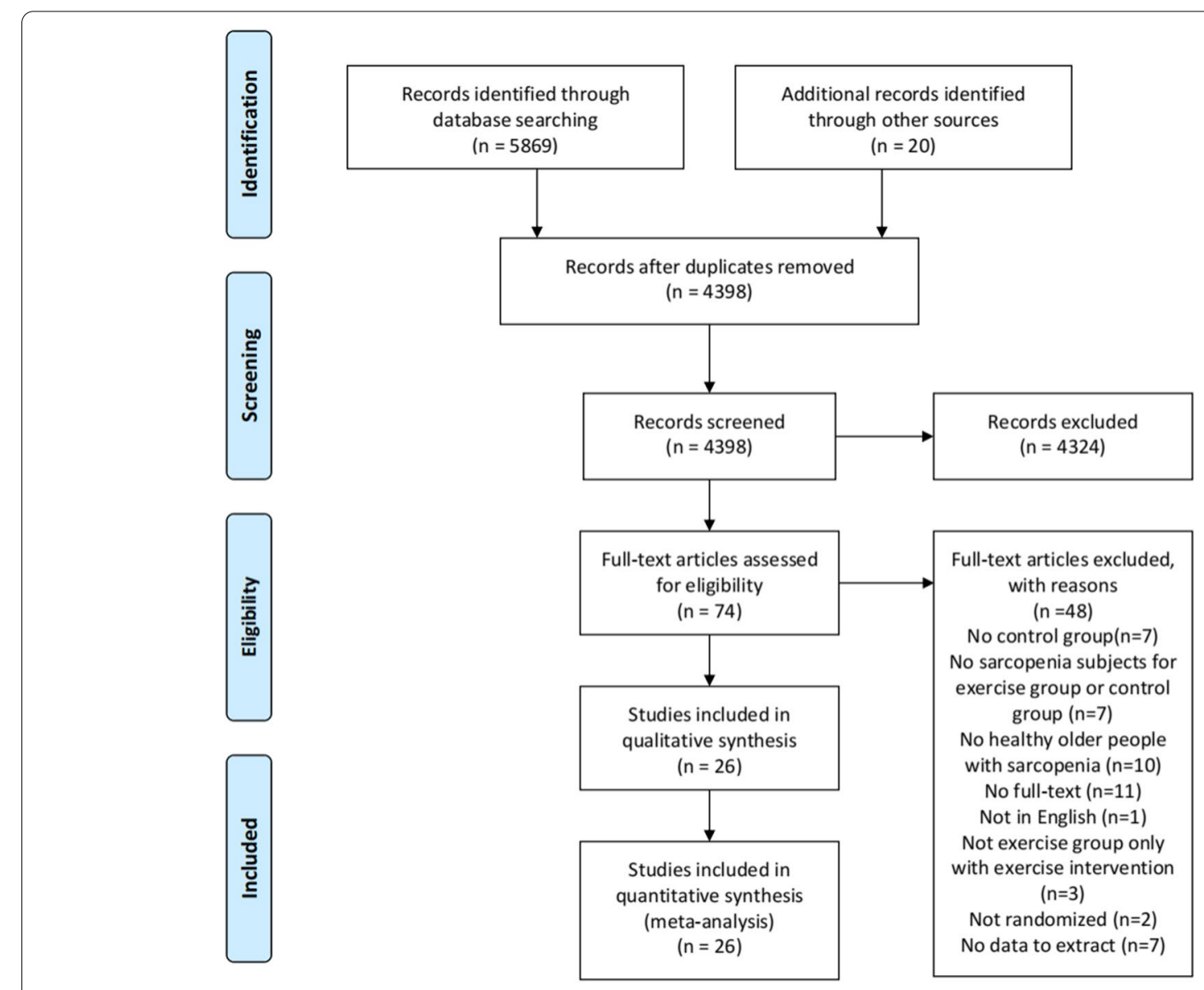

Fig. 1 The flowchart for study screening and selection process according to the PRISMA guidelines 
from the systematic reviews and other reviews retrieved in the search process. After removing duplicate studies, 4386 studies remained. The study titles and abstracts then were screened to apply the inclusion and exclusion criteria. This resulted in 4319 studies being removed for failing to meet the inclusion criteria. Of the remaining 74 studies, the full text was read to apply the exclusion criteria. The final sample size was 26 studies with full text for the systematic review and meta-analysis.

\section{Study characteristics}

Tables 1, 2 and 3 shows the characteristics of the 26 studies to include the publication dates, sample size and populations studied by gender and age, sarcopenia diagnosis, intervention details of exercise mode, intensity, duration, and training movements, inclusion of a control group, and outcomes of the studies. Two studies included two exercise modes. Chen et al. [49] included RT and MT and Bellomo et al. [50] included RT and WBVT. The studies are included in the analysis for each of their exercise training modes.

\section{Publication dates and languages}

The 26 studies were published from 2012 to 2020 with four in 2020, three in 2019, three in 2018, five in 2017, six in 2016, one in 2015, three in 2013, and one in 2012. Two studies were written in Chinese and the rest were written in English.

\section{Sample size and populations studied}

A total of 1191 older people with sarcopenia were enrolled in the studies with 613 in exercise groups and 578 in the control groups. The sample sizes in the exercise groups ranged from seven to 41 subjects and the control groups ranged from eight to 39 subjects. The mean age of the subjects ranged from $60.6 \pm 2.3$ to $89.5 \pm 4.4$ [65]. 11 studies [16, 23, 33, 49, 51, 52, 54-56, 61, 65] included both genders, five [50,53, 58-60] included only males, eight [57, 62-64, 66-69] included only females, and two studies $[70,71]$ did not identify the genders.

\section{Sarcopenia diagnosis}

Six studies [16, 33, 52, 53, 55, 59] diagnosed sarcopenia using the criteria from the Asian Working Group for Sarcopenia (AWGS), seven studies [23, 56, 58, 60, 62, 64, 70] used the criteria from the European Working Group on Sarcopenia in Older People (EWGSOP), and the remaining 13 studies [49-51, 54, 57, 61, 63, 65-69, 71] did not describe their diagnostic criteria in detail, only that they used specific index values. Table 4 shows the different sarcopenia diagnostic criteria used in all included studies.

\section{Intervention details}

For the 20 RT studies [16, 23, 49, 50, 54-58, 60, 63, 66, $67,69]$,the following training movements were used: six studies $[49,50,55,58,60,67]$ used weight machines, four studies [16, 23, 56, 69] used individual body weights, one study used dumbbells [54], three studies $[54,63,66]$ used ankle/wrist weights, ten studies [33, $52,56,57,63,64,66-68,71]$ used bands, and one study [62] used weighted vests.

Twelve studies $[23,33,49,52,54,55,57,64,67,68$, $70,71]$ focused on the muscles of upper and lower limbs and nine studies $[16,50,56,58,60,62,63,66$, 69] focused on the muscles of lower limbs. The exercise intensities were distributed as follows: six studies [49, $52,54,58,60,69$ ] ranged from 60 to $80 \%$ of 1 repetition maximum (RM), one study was $60-85 \%$ of maximum theoretical force [50], ten studies [23, 33, 56, 57, 63, $64,66-68,70]$ use the Borg CR-10 scale and four studies $[16,55,62,71]$ did not describe the intensity of the training. The duration of the exercise sessions lasted 20 to $60 \mathrm{~min}$, the training frequency varied from 1 to 5 times per week and the length of the training period varied from 6 weeks to 6 months.

For WBVT, all six studies [50, 51, 53, 59, 61, 65] used a WBV machine to apply the training. The frequency of WBVT ranged from 12 to $60 \mathrm{~Hz}$ in five studies [51, $53,59,61,65]$ with one study [50] using a time-modulated sinusoidal signal up to $300 \mathrm{~Hz}$. The duration of the exercise sessions lasted of 15 to $40 \mathrm{~min}$, the training frequency varied from 3 to 5 times per week, and the length of training period varied from 3 to 8 months.

For MT, all 14 studies $[16,23,33,49,52,58,60,63$, 66-71] used a comprehensive progressive exercise program that included resistance and balance training (one study [70]), resistance and endurance training (two studies [58, 60]), resistance, balance and gait training (two studies $[63,66]$ ), resistance, balance and aerobic training (two studies [33, 71]), resistance, balance, aerobic and gait training (one study [23]), resistance and aerobic training (five studies [16, 49, 52, 67, $68]$ ), and resistance training and stretching (one study [69]). The exercise sessions lasted 60 to $90 \mathrm{~min}$, the training frequency varied from 1 to 5 times per week, and the length of training period varied from 8 weeks to 6 months.

\section{Control group}

Nineteen studies $[16,49-54,56-65,69,70]$ required subjects to maintain their usual daily lifestyles without any exercise interventions, subjects in six studies [23, $33,55,66-68]$ received an educational course and subjects in one study [71] received a relaxation exercise program. 


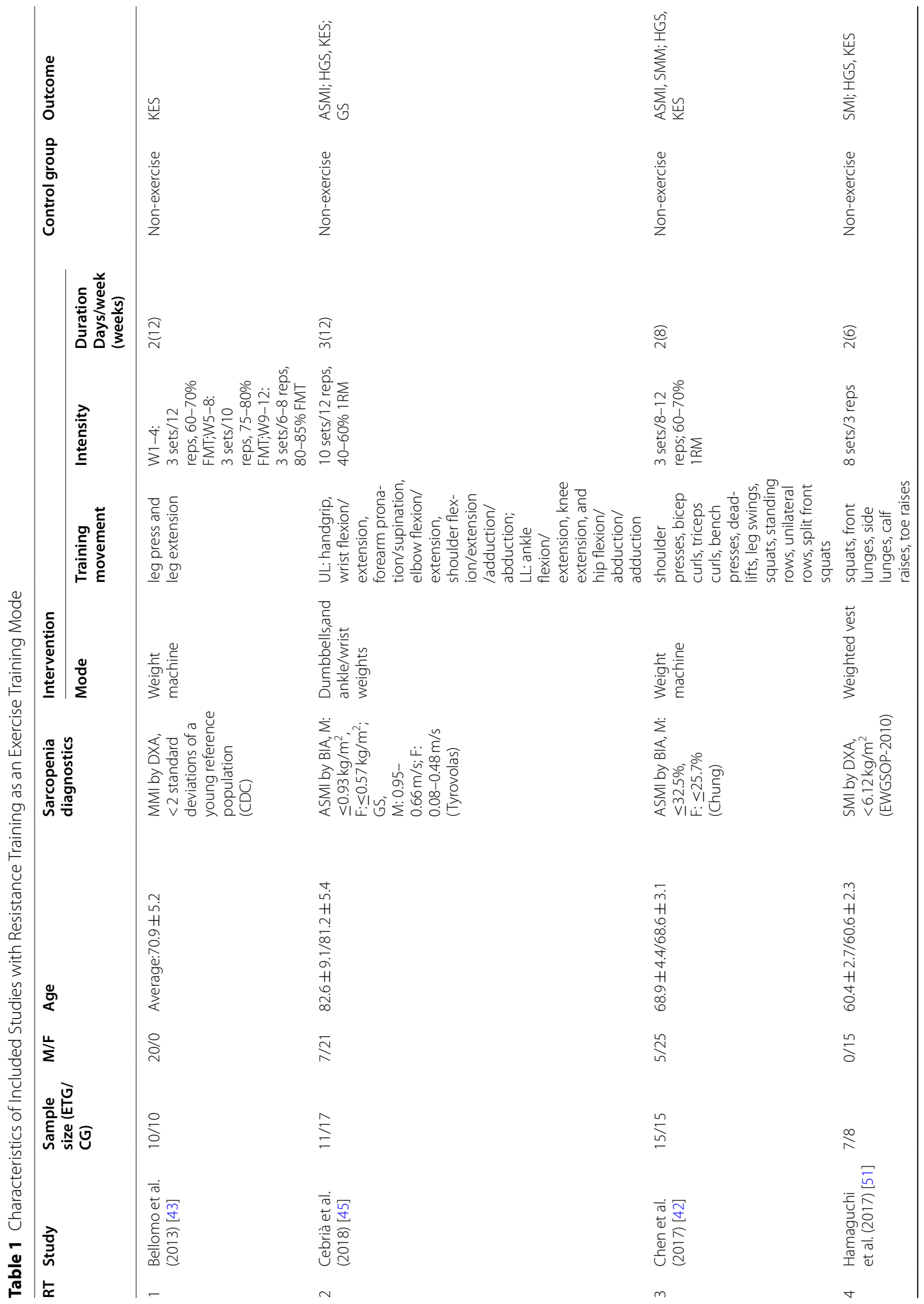




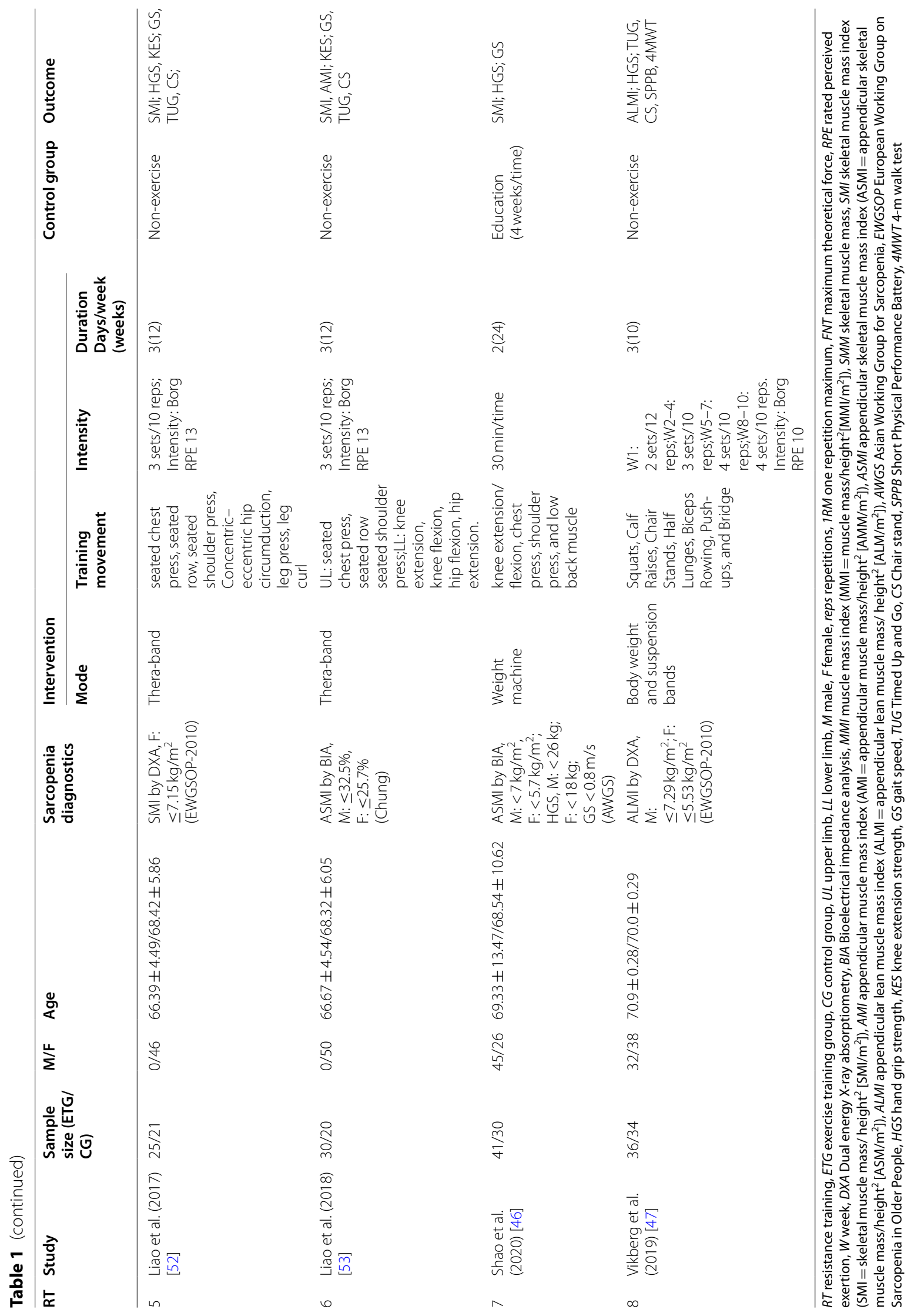




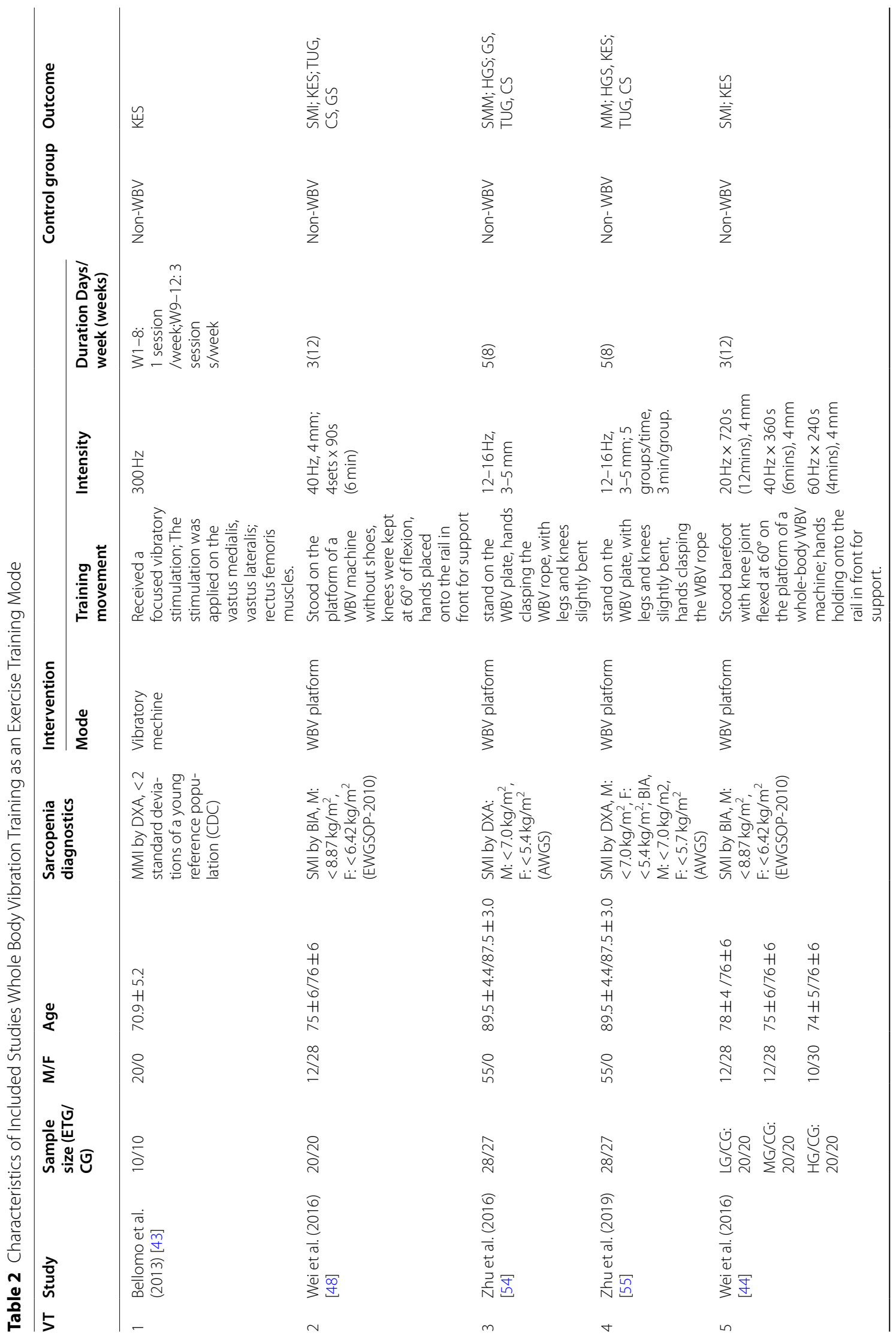




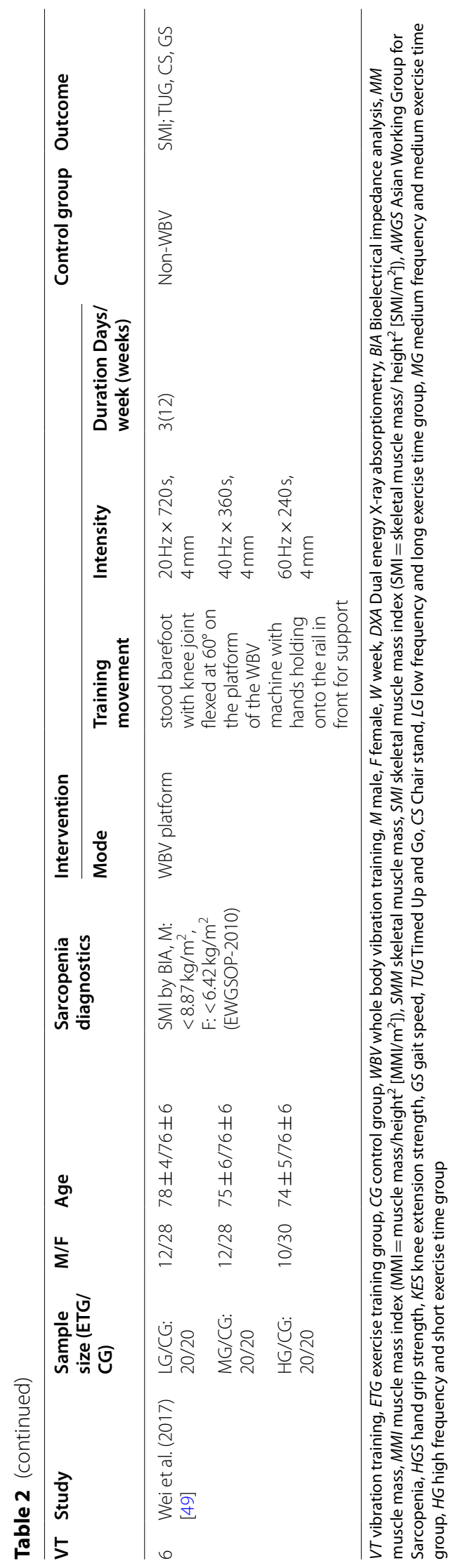




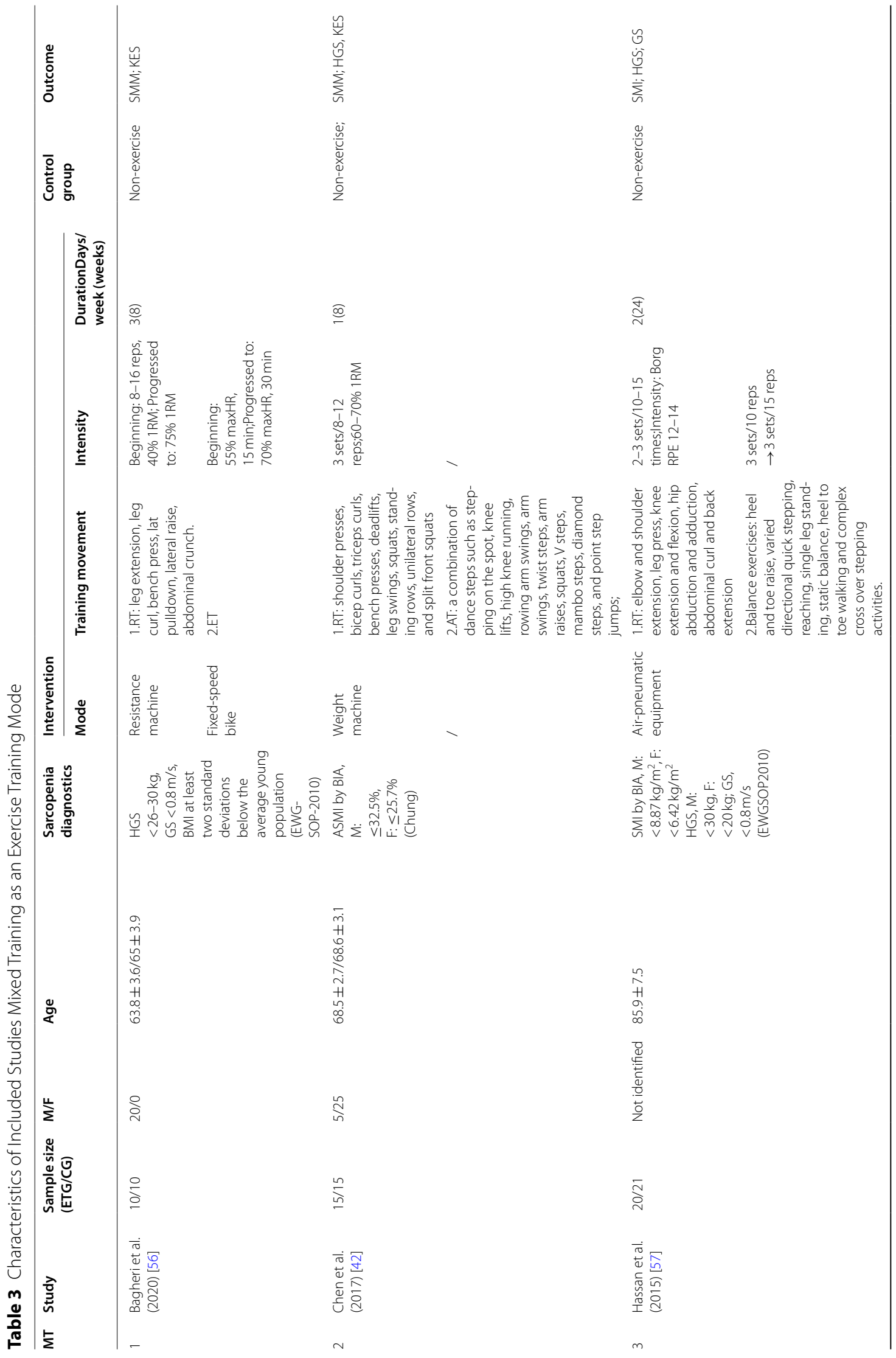




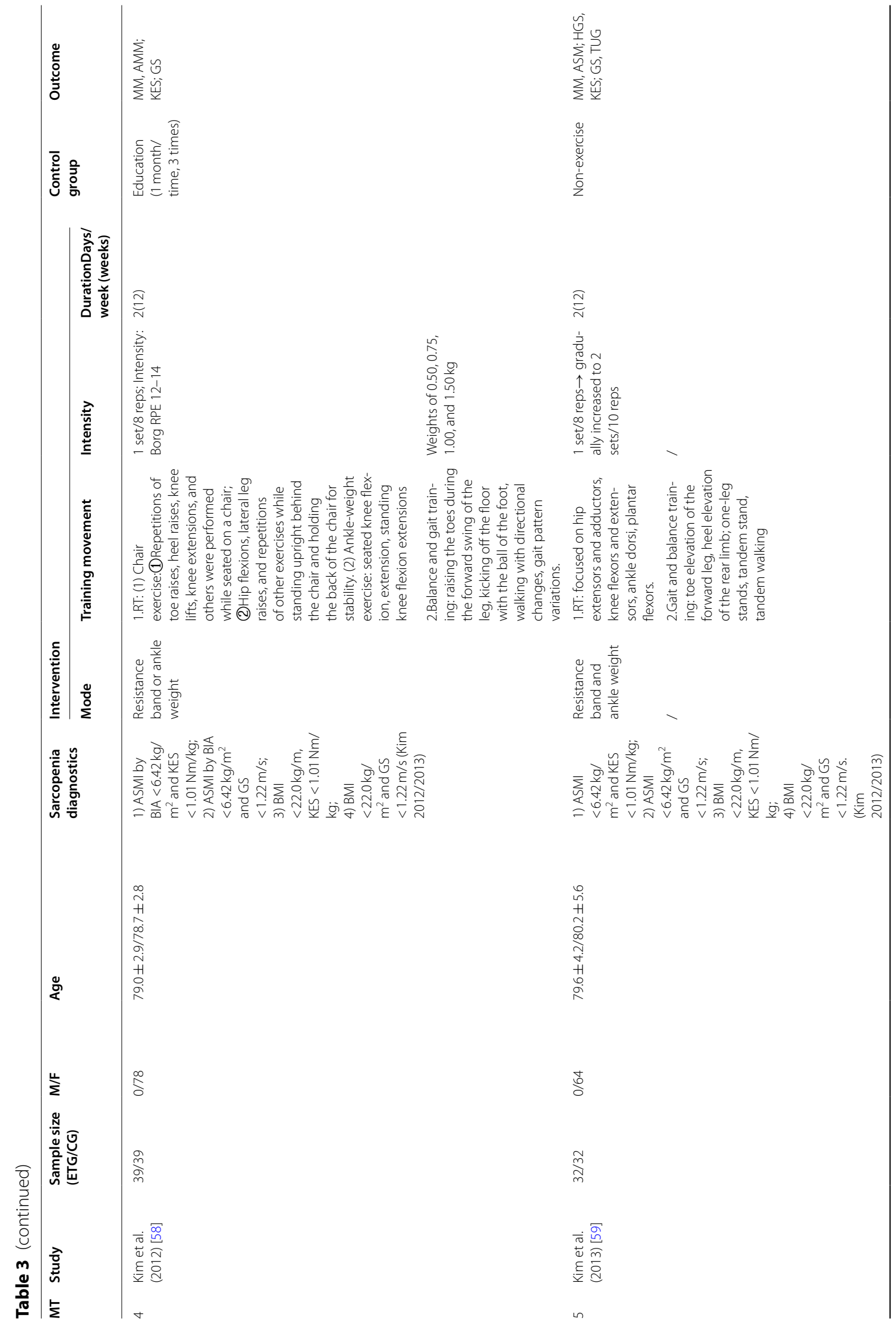




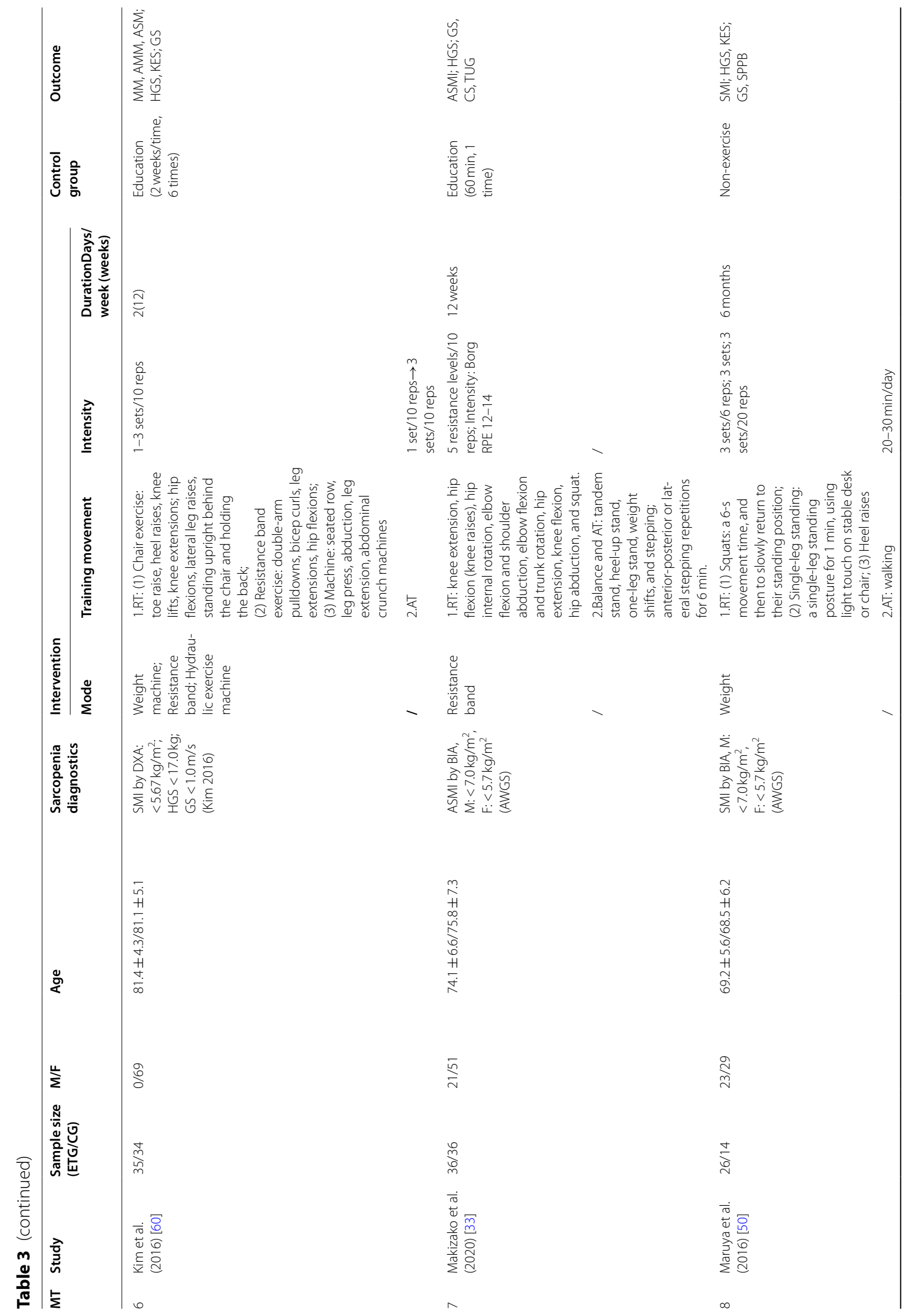




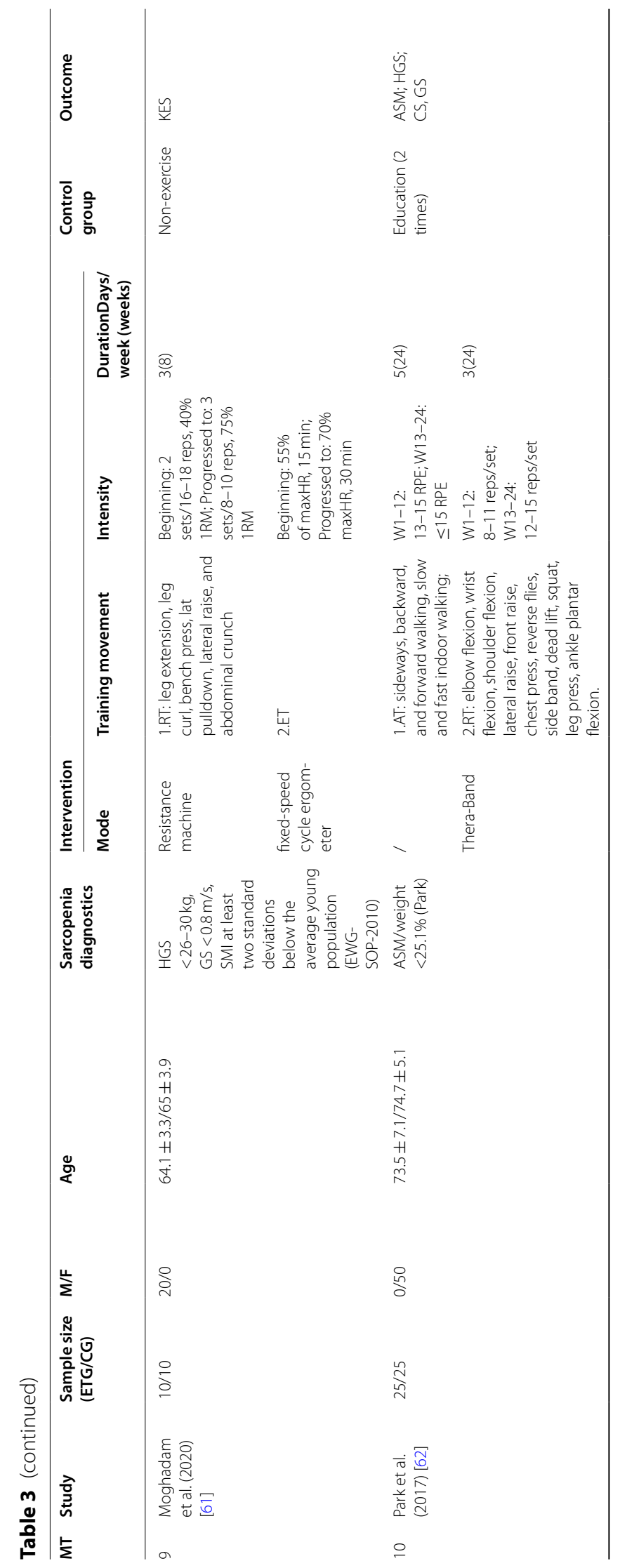




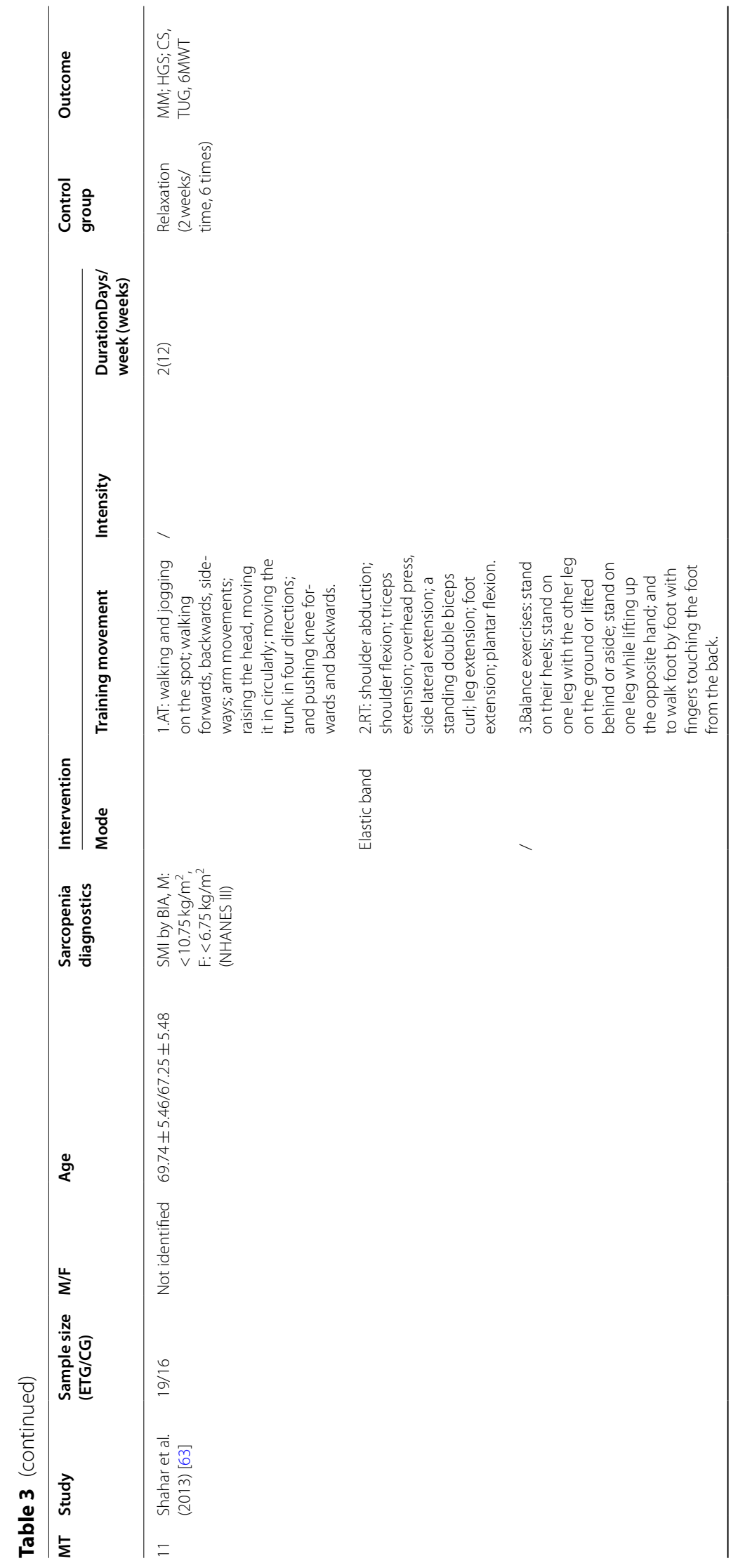




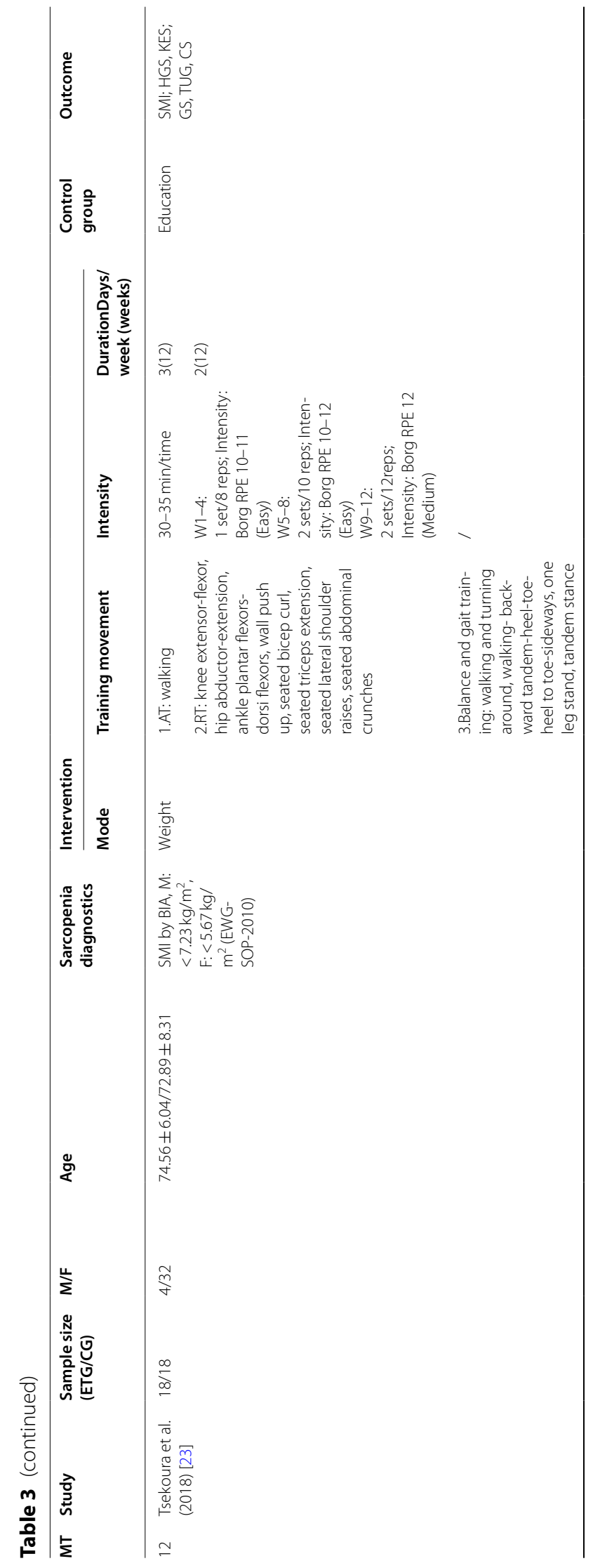




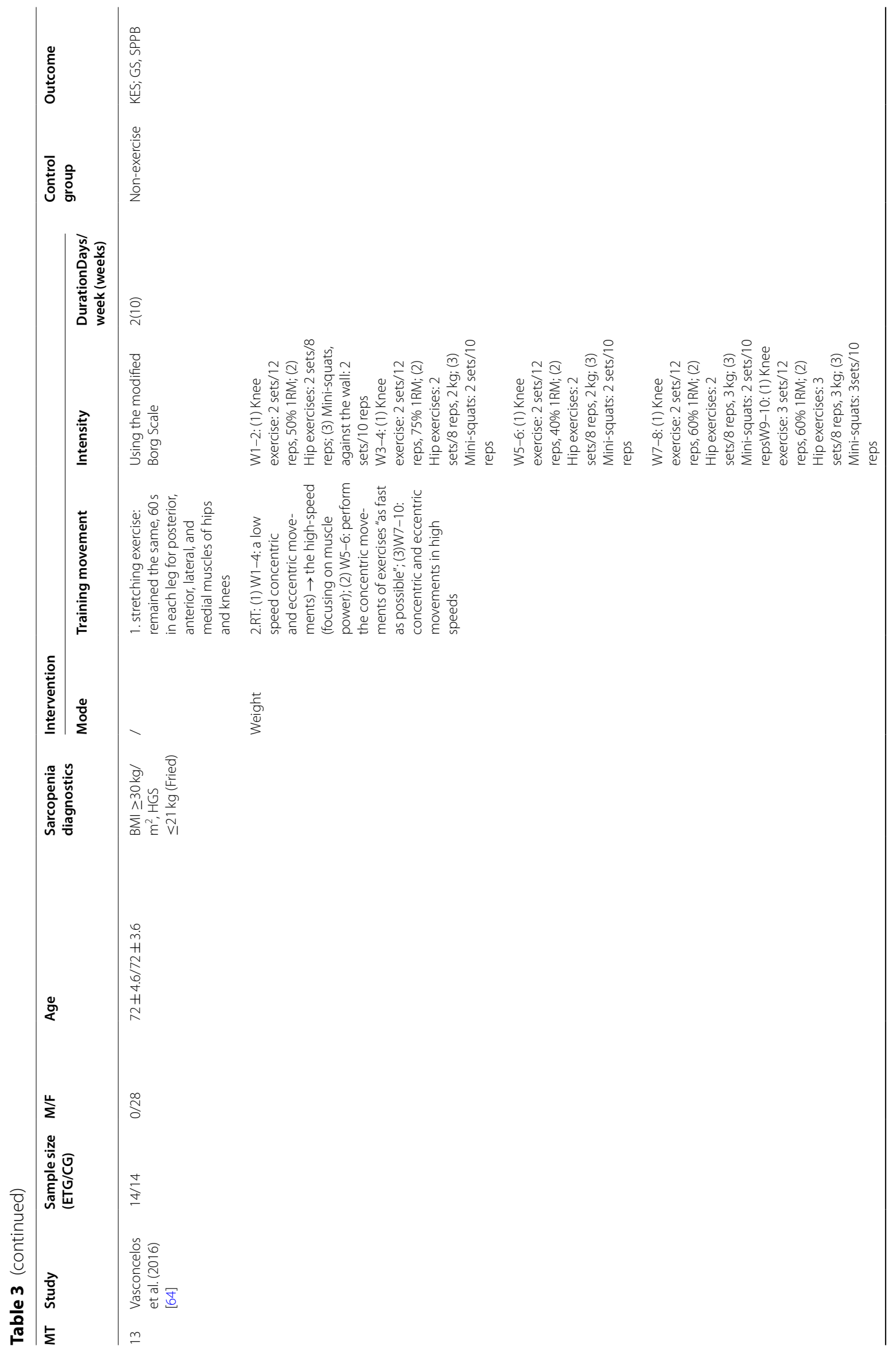




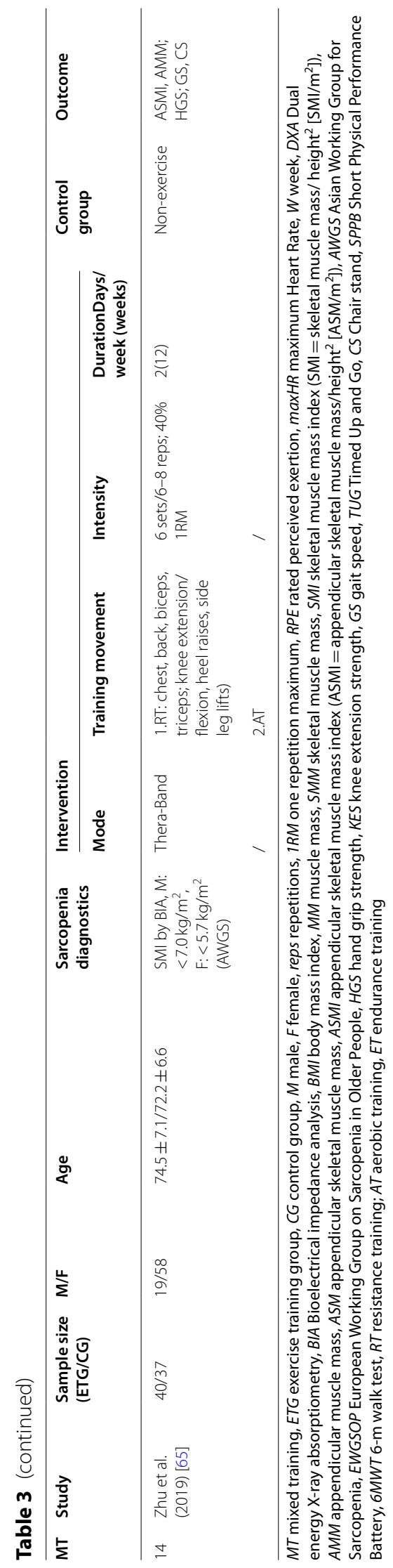


Table 4 Different indicators and cut-off points in defining sarcopenia

\begin{tabular}{|c|c|c|c|c|}
\hline \multirow[t]{2}{*}{ Diagnosis Criteria } & \multirow[t]{2}{*}{ Target district } & \multicolumn{3}{|l|}{ Cut-off points } \\
\hline & & Muscle mass & Muscle strength & Physical performance \\
\hline AWGS [72] & countries from Asia & $\begin{array}{l}\text { ASMI by DXA: }\left(\mathrm{M}:<7.0 \mathrm{~kg} / \mathrm{m}^{2}\right. \\
\left.\text { F: }<5.4 \mathrm{~kg} / \mathrm{m}^{2}\right) ; \text { Or ASMI by BIA: } \\
\left(\mathrm{M}:<7.0 \mathrm{~kg} / \mathrm{m}^{2}, \mathrm{~F}:<5.7 \mathrm{~kg} / \mathrm{m}^{2}\right)\end{array}$ & HGS: (M:<28 kg, F:<18kg) & $\begin{array}{l}\mathrm{GS}:<1.0 \mathrm{~m} / \mathrm{s} ; \text { Or } 5-\mathrm{STS} \geq 12 \mathrm{~s} ; \text { Or } \\
\text { SPPB: } \leq 9\end{array}$ \\
\hline EWGSOP-2010 [73] & countries from Europe & $\begin{array}{l}\text { ASMI by DXA: }(\mathrm{M}:<7.23 \mathrm{~kg} / \\
\left.\mathrm{m}^{2}, \mathrm{~F}:<5.67 \mathrm{~kg} / \mathrm{m}^{2}\right) ; \text { or ASMI } \\
\text { by BIA: }\left(\mathrm{M}:<8.87 \mathrm{~kg} / \mathrm{m}^{2}, \mathrm{~F}:\right. \\
\left.<6.42 \mathrm{~kg} / \mathrm{m}^{2}\right)\end{array}$ & HGS: (M: <30 kg, F: <20 kg) & GS: $<1.0 \mathrm{~m} / \mathrm{s}$; or SPPB: $\leq 8$ \\
\hline CDC [74] & New Mexico & $\begin{array}{l}\mathrm{ASM}^{*}<2 \text { standard deviations } \\
\text { of a young reference popula- } \\
\text { tion }\end{array}$ & / & / \\
\hline $\begin{array}{l}\text { National Center for } \\
\text { Health Statistics [75] }\end{array}$ & NHANES III & $\begin{array}{l}\text { SMI by BIA, M: }<10.75 \mathrm{~kg} / \mathrm{m}^{2} \text {, F: } \\
<6.75 \mathrm{~kg} / \mathrm{m}^{2}\end{array}$ & / & / \\
\hline Park [62] & Busan City, South Korea & ASM2/weight <25.1\% & / & / \\
\hline Chung [76] & Korea & $\begin{array}{l}\text { ASMI2 by BIA, } \mathrm{M}: \leq 32.5 \%, \mathrm{~F}: \\
\leq 25.7 \%\end{array}$ & / & / \\
\hline $\operatorname{Kim} 2012 / 2013[58,59]$ & Tokyo & $\begin{array}{l}\text { ASMI3 by BIA }<6.42 \mathrm{~kg} / \mathrm{m}^{2} \\
\mathrm{BMI}<22.0 \mathrm{~kg} / \mathrm{m}\end{array}$ & $\mathrm{KES}<1.01 \mathrm{Nm} / \mathrm{kg}$ & $\mathrm{GS}<1.22 \mathrm{~m} / \mathrm{s}$ \\
\hline Kim 2016 [60] & Tokyo & SMI by DXA $<5.67 \mathrm{~kg} / \mathrm{m}^{2}$ & $\mathrm{HGS}<17.0 \mathrm{~kg}$ & $\mathrm{GS}<1.0 \mathrm{~m} / \mathrm{s}$ \\
\hline Tyrovolas [77] & $\begin{array}{l}\text { countries from Asia, Africa, } \\
\text { Europe, and Latin America }\end{array}$ & $\begin{array}{l}\text { ASMI4 by BIA, M: } \leq 0.93 \mathrm{~kg} / \mathrm{m}^{2} \\
\mathrm{~F}: \leq 0.57 \mathrm{~kg} / \mathrm{m}^{2}\end{array}$ & HGS: (M: <30 kg, F: <20 kg) & $\begin{array}{l}\text { GS: (M: } 0.95-0.66 \\
\mathrm{~m} / \mathrm{s} ; \mathrm{F}: 0.08-0.48 \mathrm{~m} / \mathrm{s})\end{array}$ \\
\hline Fried [78] & $\begin{array}{l}\text { countries from African } \\
\text { American }\end{array}$ & $\begin{array}{l}\text { baseline: >10lbs lost uninten- } \\
\text { tionally in prior year }\end{array}$ & $\begin{array}{l}\text { HGS: lowest 20\% (by gen- } \\
\text { der, body mass index) }\end{array}$ & $\begin{array}{l}\text { walking time/15ft: slowest 20\% } \\
\text { (by gender, height) }\end{array}$ \\
\hline
\end{tabular}

AWGS Asian Working Group for Sarcopenia, EWGSOP European Working Group on Sarcopenia in Older People, CDC Centers for Disease Control and Prevention, NHANES III Third National Health and Nutrition Examination Survey, M male, F female, ASMI appendicular skeletal muscle mass index (ASMI=appendicular skeletal muscle mass/height $\left.{ }^{2}\left[\mathrm{ASM} / \mathrm{m}^{2}\right]\right), A S M I 2=$ appendicular skeletal muscle mass $/$ weight ${ }^{2 *} 100 \%\left[\mathrm{ASM} / \mathrm{kg}^{2}\right], A S M / 3$ appendicular skeletal muscle mass index $\left(\mathrm{ASMI}=\right.$ appendicular skeletal muscle mass $\left./ \mathrm{height}^{2}\left[\mathrm{ASM} / \mathrm{m}^{2}\right]\right), A S M I 4=[0.244$ (weight) +7.8 (height) +6.6 (sex)-0.098(age) + race-3.3]/body mass index (ASM/BMI), SMI skeletal muscle index; $\mathrm{ASM}^{*}=0.2487$ (weight) +0.0483 (height)-0.1584(hip circumference) +0.0732 (grip strength) +2.5843 (sex) $+5.8828, A S M 2$ appendicular skeletal muscle mass, DXA Dual energy X-ray absorptiometry, BIA Bioelectrical impedance analysis, HGS handgrip strength, KES knee extension strength, GS gait speed, 5-STS 5-chair sit to stand test, SPPB Short Physical Performance Battery, TUG Timed Up and Go

\section{Outcome measures}

Some studies measured outcomes that were not analyzed in this study. Among the studies with outcomes of interest, 17 studies [16, 23, 49, 50, 54, 57, 59-67, 69] measured muscle strength by KES, 11 studies [23, 33, 51, 53, 56, 57, $59,61,63,64,71]$ measured TUG time, 17 studies [16, 23, $33,51-55,57,61,63,64,66-70]$ measured GS, 11 studies $[23,33,52,53,56,57,59,61,64,68,71]$ measured CS times.

\section{Study quality}

The PEDro scores of each study for the quality assessment are shown in Table 5. The score ranges from 0 to 10 with a mean quality score of 6.36. Among the studies, four studies scored 8 points, eight studies scored 7 points, eleven studies scored 6 points and three studies scored 5 points. The quality assessment of the study consisted of 11 criteria: 25 studies reported the random allocation, and only one study performed non-randomized controlled trial. The baseline was similar in all studies. Seventeen studies [23, 33, 51-57, 59, 61, 63-67, 69] reported a concealed allocation. For blinding, three studies [33, 64, $70]$ used subject blinding, five studies [54, 57, 63, 64, 66] used therapists blinding and 11 studies $[49,50,52,54,56$, 62-64, 66, 68, 69] used assessor blinding. Twenty-three studies [16, 23, 33, 50-53, 55-62, 64-71] reported > 85\% of the subjects performing at least one primary outcome measure, ten studies $[23,33,53,55,58-60,63,68,71]$ reported the data from intention-to-treat analysis, 25 studies [23, 33, 52, 54, 56-60, 62-64, 66-70] performed group comparison and 23 studies [23, 33, 52, 54, 56-63, 66-70] performed point measures.

Details about the risk of bias of the included studies are shown in Fig. 2. Figure 2a is a plot of the distribution of studies at low-, unclear-, or high risk of bias based on the Cochrane risk-of-bias tool and Fig. $2 b$ is a summary table for risks of bias in each study. For the random sequence generation assessment, the risk of bias was unclear in eight studies $[16,33,49,50,58,60$, $62,68]$ and the risk of bias was high in one study [71]. For the allocation concealment assessment, the risk of bias was unclear in three studies $[60,62,68]$ and the risk of bias was high in six studies $[16,33,49,50,58$, 71]. For the participants and personnel assessment, the risk of bias was low in six studies [51, 58, 61, 64, 65, 69]. For the blinding of outcomes assessment, the risk of 


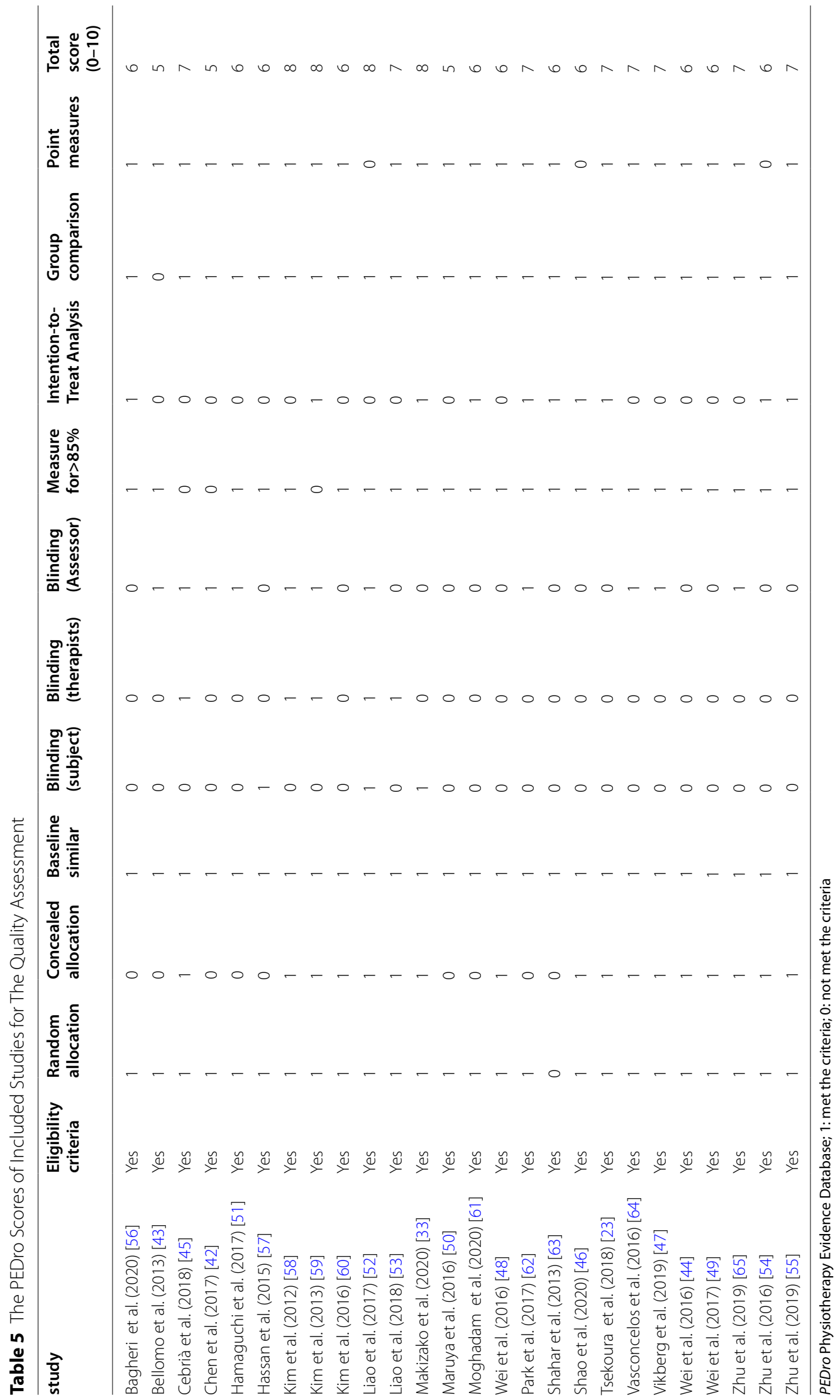




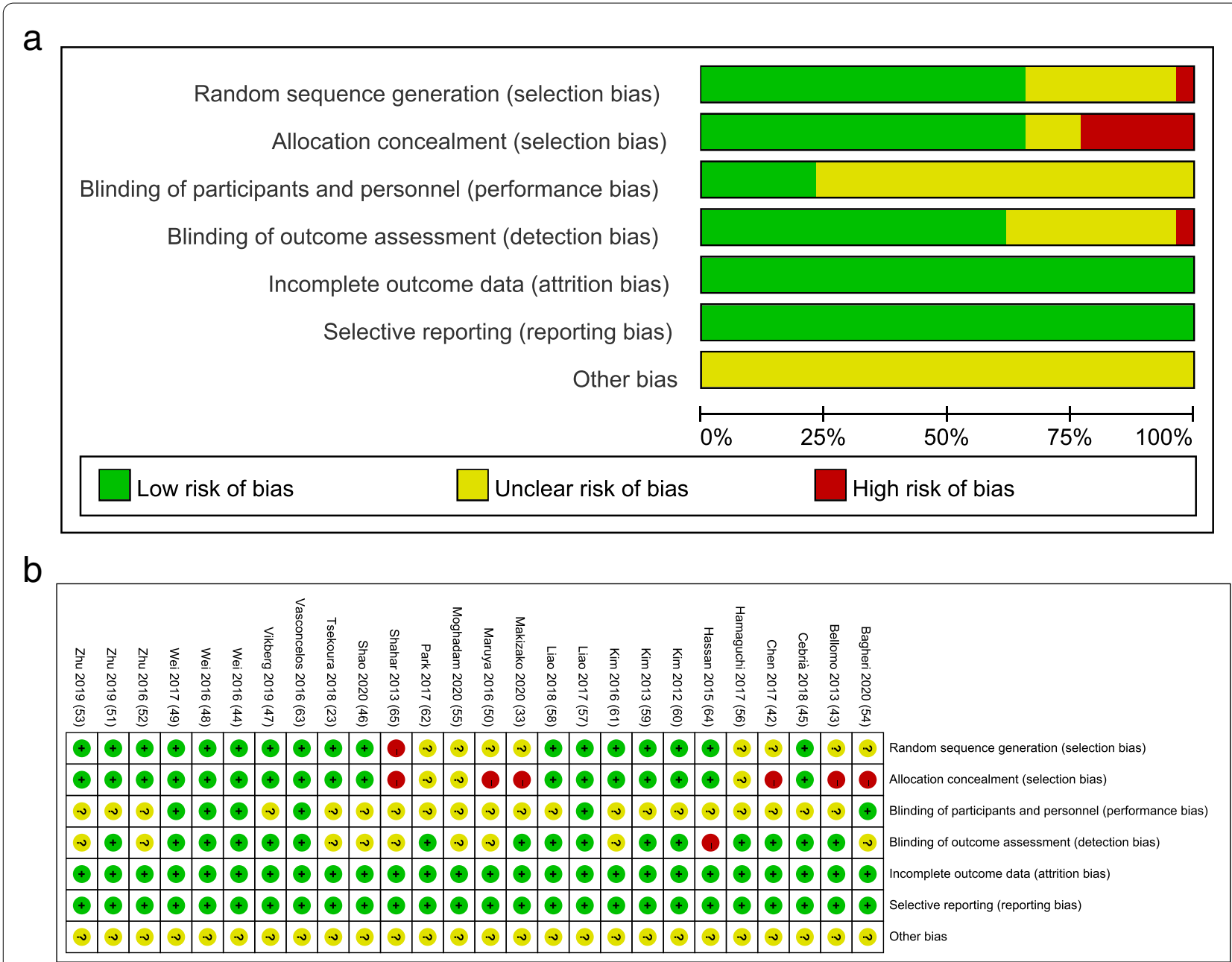

Fig. 2 Assessment of risk of bias based on the Cochrane risk-of-bias tool. a Percent of studies with categories for risk of bias; $\mathbf{b}$ Summary for the risk of bias in each study

bias was unclear in nine studies $[16,23,53,55,58-60$, $67,71]$ and the risk of bias was high in one study [70]. There were no unclear or high risk of bias observed for the incomplete outcome data assessment and the selective reporting assessment. The other bias risk items were not identified and were rated as unclear for all 26 studies.

The overall certainty of evidence across studies of the outcomes was performed in Table 6. After rating overall studies by the GRADE assessment, we found that the level of evidence was generally low. The possible reasons for this result are as follows: included assessor-blinding, concealed allocation, heterogeneity, length of followup and etc. Using the GRADE criteria, all these RCTs and non-randomised interventional studies showed a low level of evidence in KES as an indicator for muscle strength, and a low level of evidence in physical performance (TUG times, GS and CS times).

\section{Outcomes \\ Effects of different exercise training modes for sarcopenia on KES}

Figure 3 is a forest plot of the subgroup analyses of 17 [16, $23,49,50,54,57-67,69]$ studies with KES as an outcome for sarcopenia based on different exercise training modes. Collectively, 415 subjects were in exercise group and 394 subjects were in control group. Among subgroup analysis of the exercise modes, 6 studies [49, 50, 54, 57, 62, 64] used RT, 4 studies $[50,59,61,65]$ used WBVT training and 9 studies [16, 23, 49, 58, 60, 63, 66, 67, 69] used MT. With all exercise modes combined, the exercise group showed a significant increase in KES scores compared with the control group (SMD $=0.86,95 \% \mathrm{CI}: 0.55$ to $1.16, p<0.00001$, $\mathrm{I}^{2}=75 \%$ ). In subgroup analysis, $\mathrm{RT}$ and MT resulted in significant increases in KES scores compared to the control group (RT, $\mathrm{SMD}=1.36,95 \% \mathrm{CI}: 0.71$ to $2.02, p<0.0001$, $\mathrm{I}^{2}=72 \%$; MT, SMD $=0.62$, $95 \%$ CI: 0.29 to $0.95, p=0.0002$, 


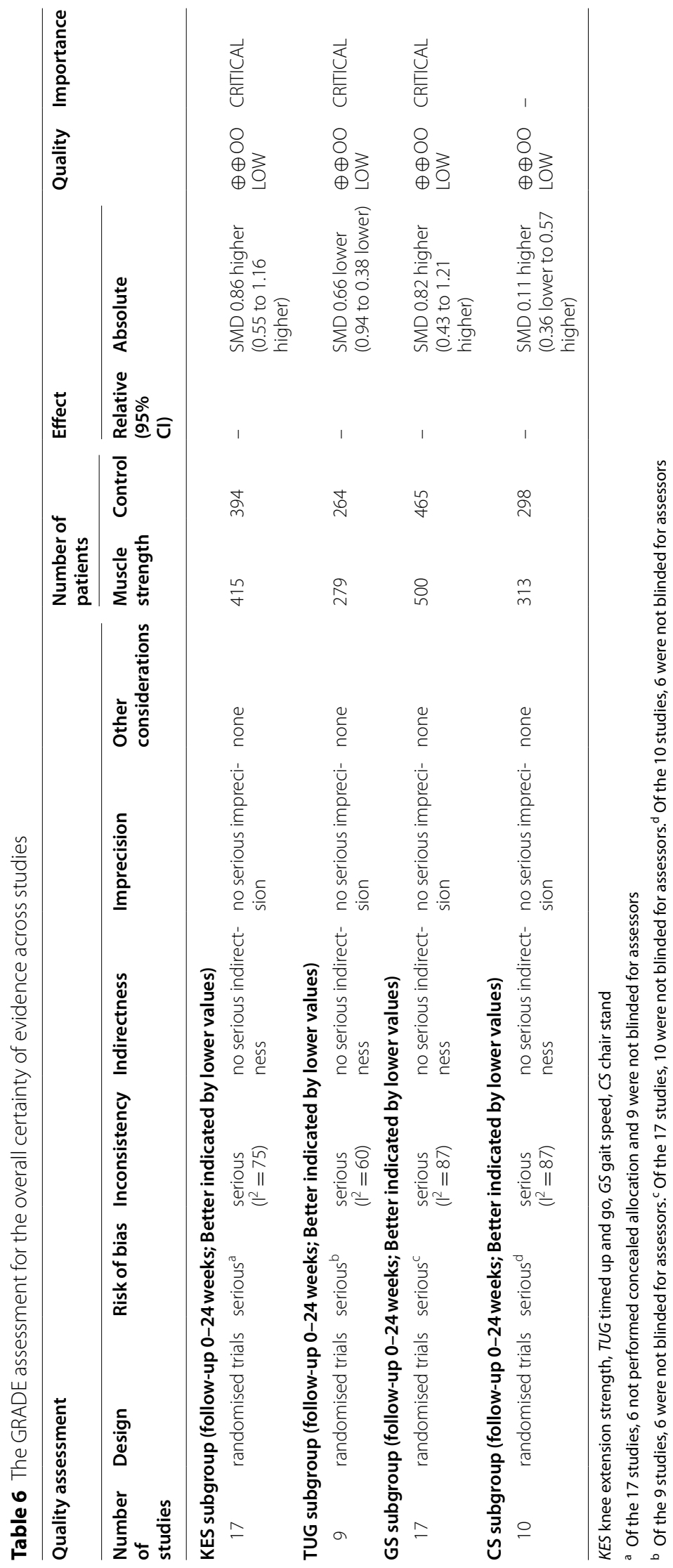




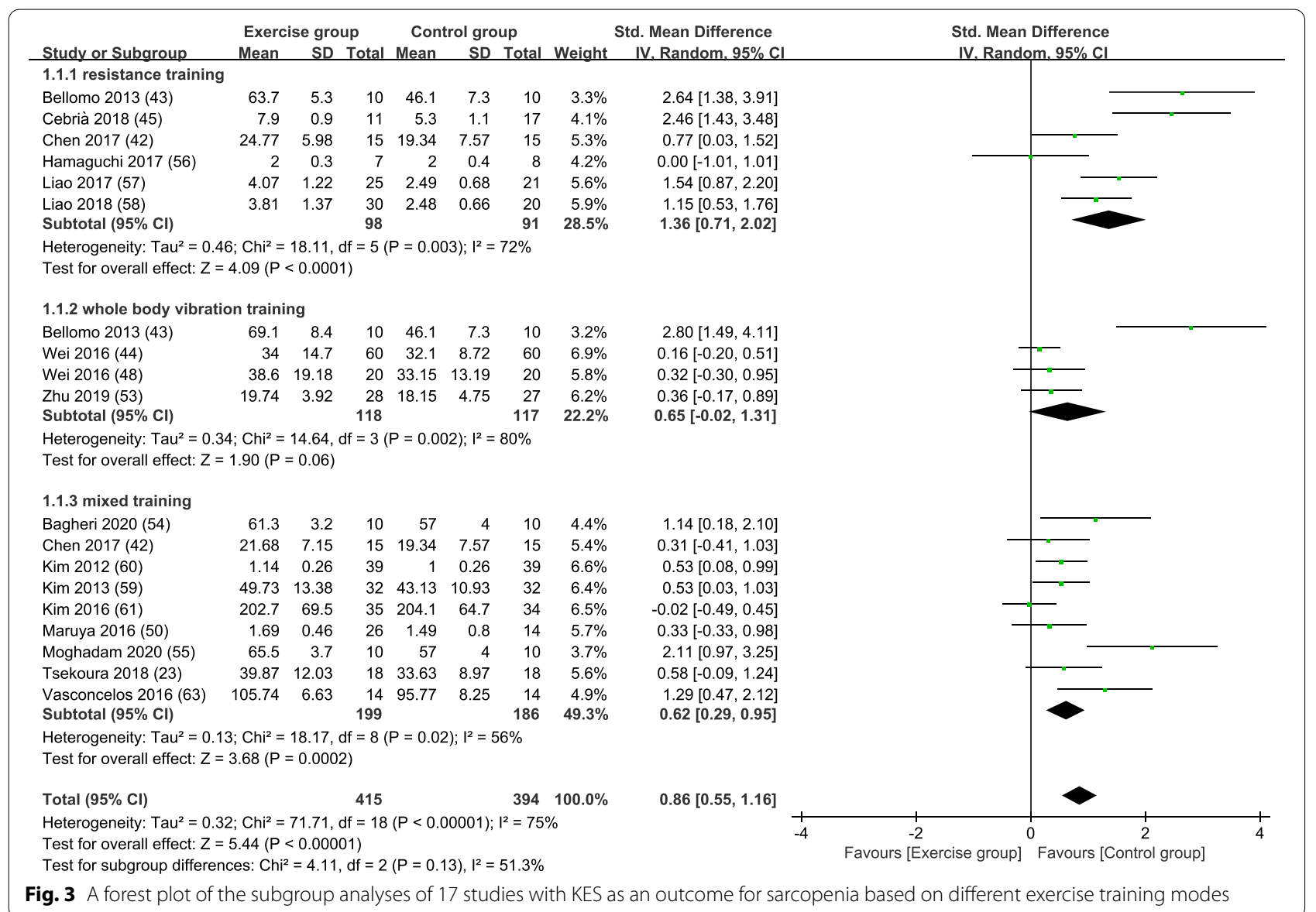

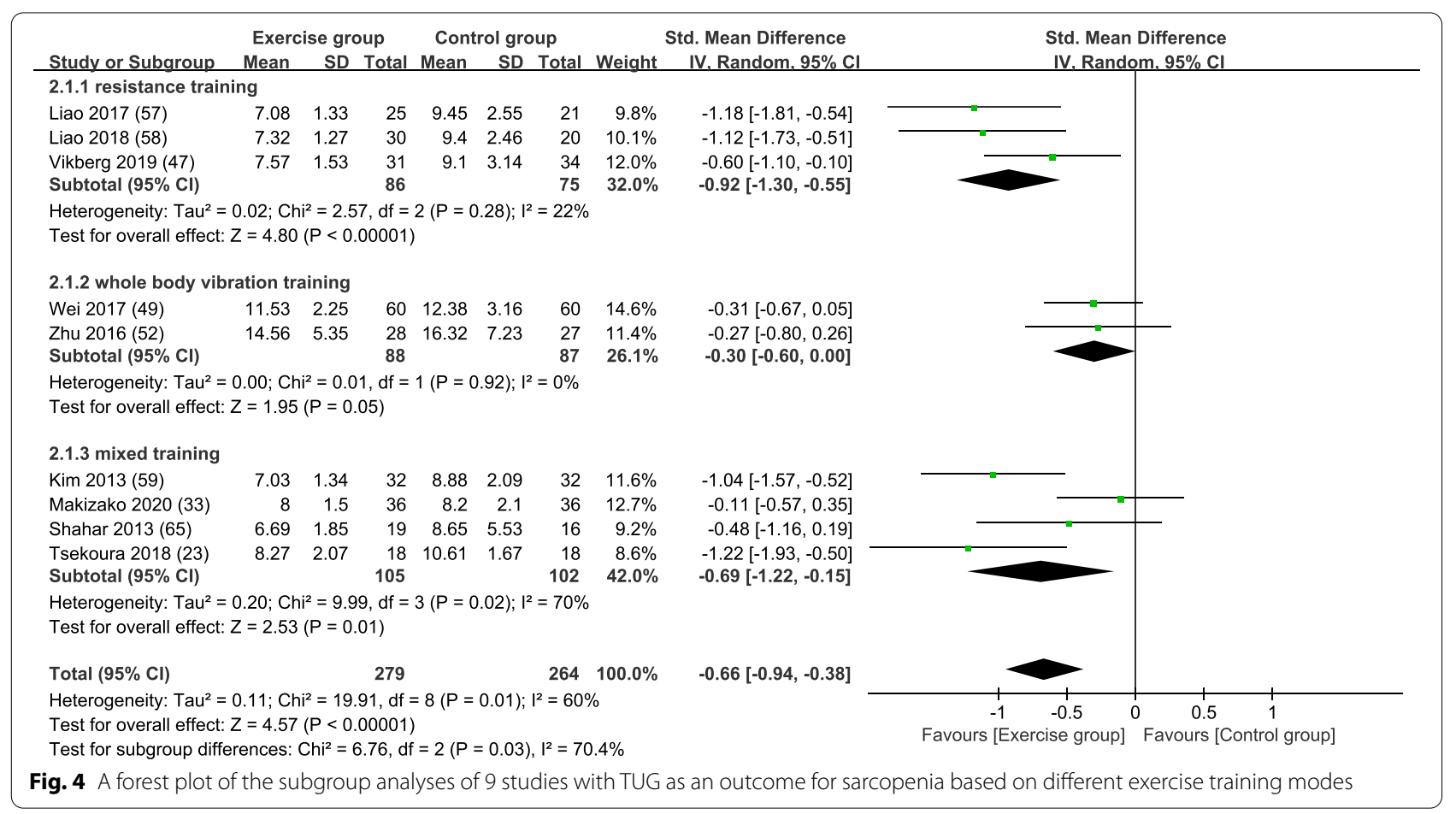




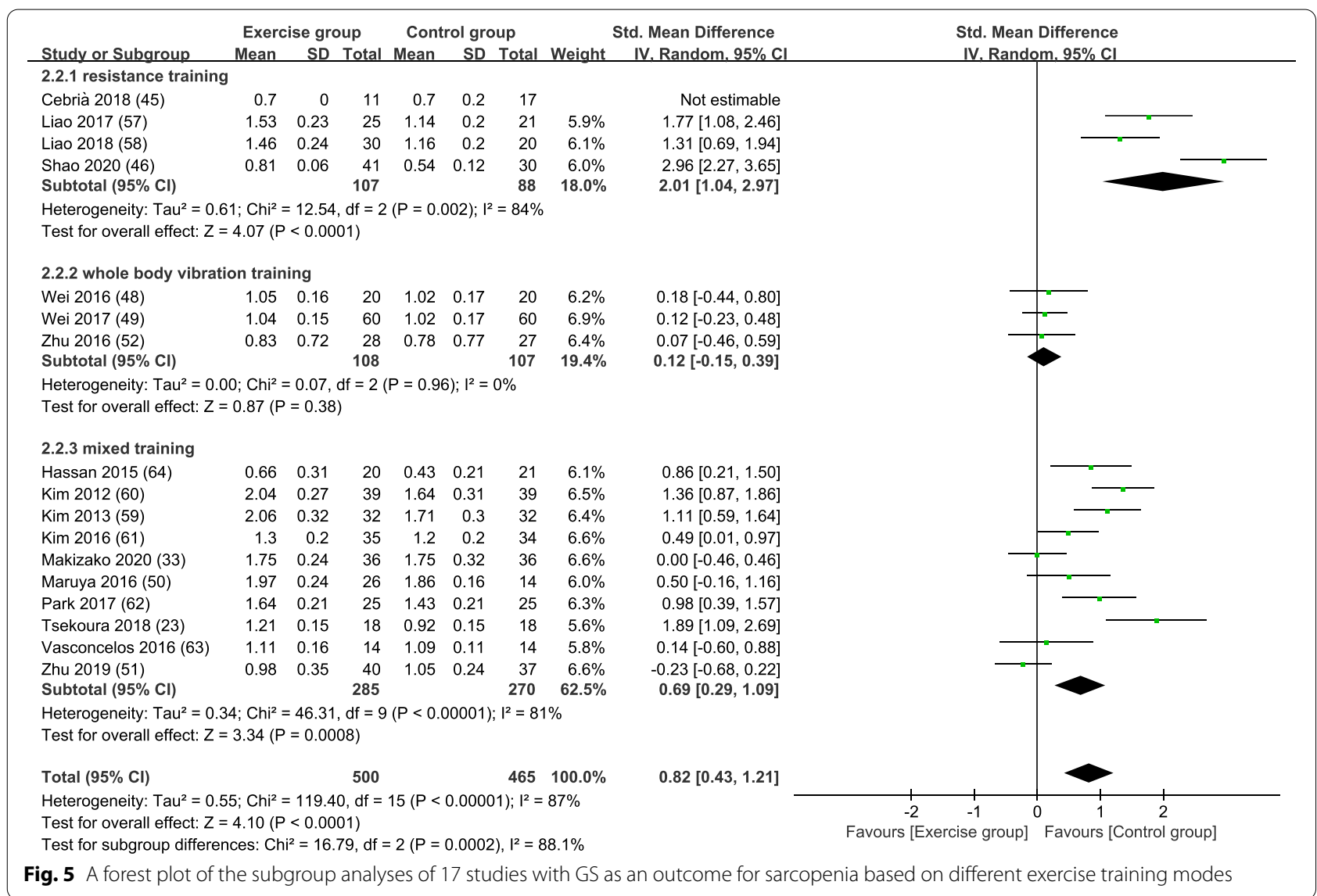

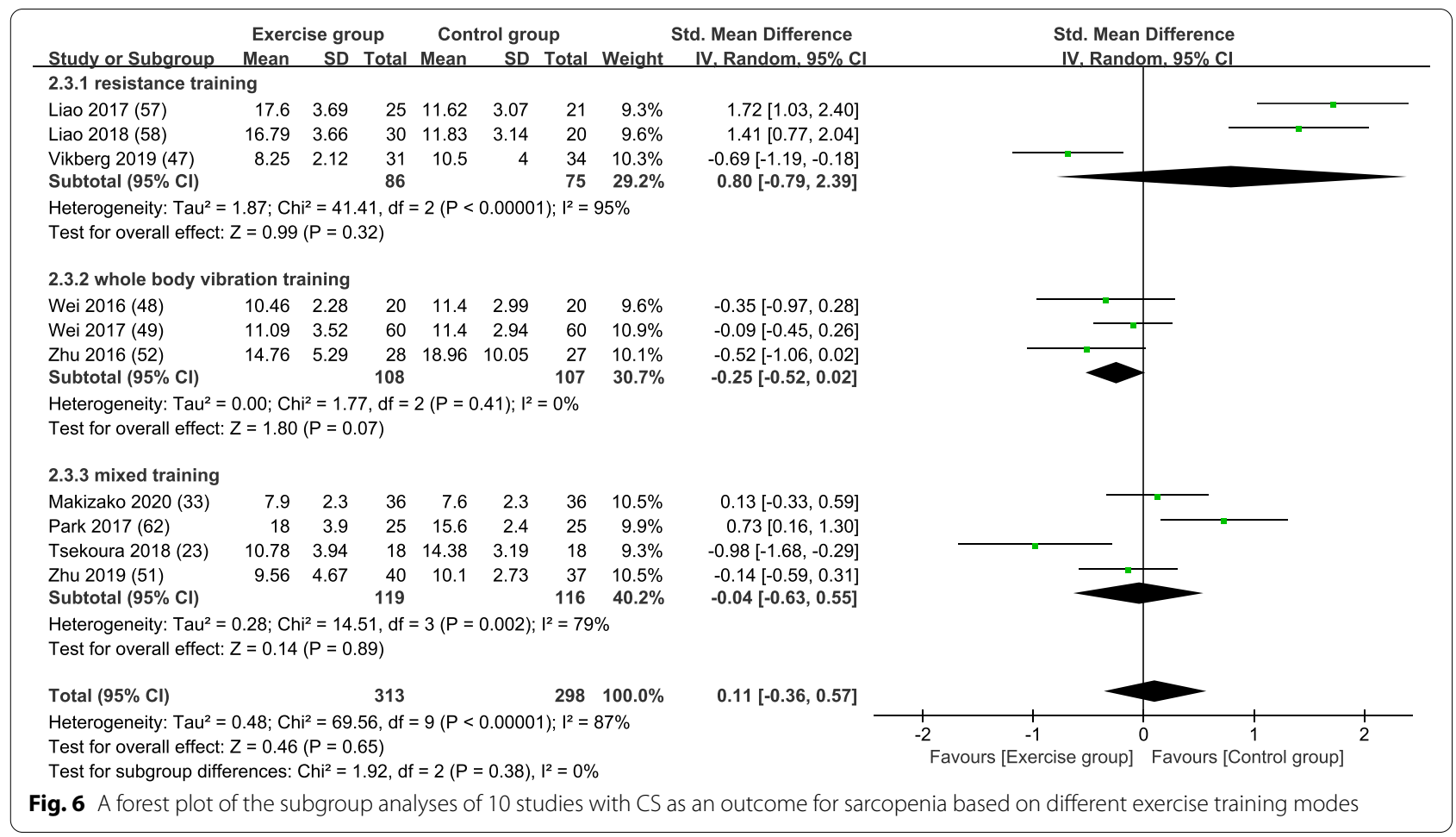


$\mathrm{I}^{2}=56 \%$ ). WBVT resulted in no significant difference in KES scores between the exercise and control groups $\left(\mathrm{SMD}=0.65,95 \% \mathrm{CI}:-0.02\right.$ to $\left.1.31, p=0.06, \mathrm{I}^{2}=80 \%\right)$.

\section{Effects of different exercise training modes for sarcopenia on physical performance}

Nineteen studies [16, 23, 33, 54-57, 61, 63, 64, 66-71] assessed the effects of exercise training on the three main outcomes of TUG, GS, and CS.

Figure 4 is a forest plot of the subgroup analyses of 9 studies (3 RT [56, 57, 64], 2 WBVT [51, 53], and $4 \mathrm{MT}$ $[23,33,63,71])$ with TUG as an outcome for sarcopenia based on different exercise training modes. Collectively, 279 subjects were in the exercise group and 264 subjects were in the control group. With all exercise modes combined, the exercise group showed a significant increase in TUG times compared with the control group (SMD $=-0.66,95 \% \mathrm{CI}:-0.94$ to -0.38 , $\left.p<0.00001, \mathrm{I}^{2}=60 \%\right)$. In subgroup analysis, all training modes showed a significant increase in TUG times compared with the control group (RT, $\mathrm{SMD}=-0.92$, 95\% CI: -1.30 to $-0.55, p<0.00001, \mathrm{I}^{2}=22 \%$; WBVT, $\mathrm{SMD}=-0.30,95 \% \mathrm{CI}:-0.60$ to $0.00, p=0.05, \mathrm{I}^{2}=0 \%$; and MT, SMD $=-0.69,95 \% \mathrm{CI}:-1.22$ to $-0.15, p=0.01$, $\left.\mathrm{I}^{2}=70 \%\right)$.

Figure 5 is a forest plot of the subgroup analyses of 17 studies with GS as an outcome for sarcopenia based on different exercise training modes. Of the 17 studies in this subgroup, 4 studies applied RT [54, 55, 57, 64], 3 studies applied WBVT [51, 53, 61] and 10 studies applied MT. [16, 23, 33, 52, 63, 66-70] Collectively, 500 subjects were in exercise group and 465 were in the control group. With all exercise modes combined, the exercise group showed a significant increase in GS compared with the control group (SMD $=0.82,95 \%$ CI: 0.43 to $\left.1.21, p<0.0001, \mathrm{I}^{2}=87 \%\right)$. In subgroup analysis, $\mathrm{RT}$ and MT showed a significant increase in GS compared with the control group (RT, SMD $=2.01,95 \% \mathrm{CI}$ : 1.04 to 2.97, $p<0.0001, \mathrm{I}^{2}=84 \%$; MT, SMD $=0.69,95 \%$ CI: 0.29 to $1.09, p=0.0008, \mathrm{I}^{2}=81 \%$ ). WBVT resulted in no significant difference in GS between the exercise and control groups (SMD $=0.12,95 \% \mathrm{CI}:-0.15$ to $0.39, p=0.38$, $\mathrm{I}^{2}=0 \%$ ).

Figure 6 is a forest plot of the subgroup analyses of 10 studies (3 RT [56, 57, 64], 3 WBVT [51, 53, 61], 4 MT $[23,33,52,68]$ ) with CS as an outcome for sarcopenia based on different exercise training modes. Collectively, 313 subjects were in the exercise group and 298 were in the control group. With all exercise modes combined, there was no significant difference in CS times between the exercise and control group $(\mathrm{SMD}=0.11,95 \% \mathrm{CI}$ : -0.36 to $0.57, p=0.65, \mathrm{I}^{2}=87 \%$ ). Subgroup analysis showed no significant differences in CS times between the exercise and control groups for RT, WBVT, and MT (RT, SMD $=0.80,95 \% \mathrm{CI}:-0.79$ to $2.39, p=0.32$, $\mathrm{I}^{2}=95 \%$; WBVT, SMD $=-0.25,95 \% \mathrm{CI}:-0.52$ to 0.02 , $p=0.07, \mathrm{I}^{2}=0 \%$; MT, SMD $=-0.04,95 \% \mathrm{CI}:-0.63$ to $\left.0.55, p=0.89, \mathrm{I}^{2}=79 \%\right)$.

\section{Discussion}

In this study, 26 studies (25 RCTs and one non-RCT) were examined using a systematic review and metaanalysis to compare the effects of RT, WBVT, and MT on muscle strength and physical performance in older people with sarcopenia. The results of our studies showed that RT can improve KES, TUG times and GS, but not CS times; WBVT have a positive effect on TUG times, but did not have a significant effect on KES, GS and CS times; The effect of MT (combinations of exercise modes such as resistance and balance training; resistance and endurance training; resistance, balance and gait training; resistance, balance and aerobic training; resistance and aerobic training) is similar with RT, which also can improve KES, TUG times and GS, but not CS times. As observed in this study, not all exercise modes improve all aspects of muscle strength and physical performance in older people with sarcopenia. These differences show the importance to identify exercise training modes that improve aspects of muscle strength and physical performance as needed most by older people with sarcopenia [79].

So far, RT is one of the most common mode of exercises used to prevent and/or delay the progression of sarcopenia in a variety of older populations. Previous studies showed that RT can improve KES [80-82], TUG times [83] and GS [84] in older people with sarcopenia, which is consistent with the result of our study. However, CS times was not been significantly improved by RT in our study. We speculated that different RT protocols of included RT studies may be a reason why RT has no significant effect on CS in our study. Given that several studies reviewed in this systematic review and meta-analysis included squats in RT studies [49, 56, 62], it is possible that the dose of exercise need to improve sit-to-stand performance was insufficient for older people with sarcopenia. And studies by Soligon et al. [85] and Vitale et al. [86] demonstrated that RT protocols including squat movements can significantly improve CS times, which may be due to the sufficient exercise dose and increased proficiency of squat movements of older people in our study. Thus, we speculated that RT protocols including squat movements is an important factor which may produce an effective influence on CS times.

Our systematic review and meta-analysis failed to show beneficial effects of WBVT on KES, GS and CS times, but it showed improvements on TUG times in older people with sarcopenia. The result of TUG times is consistent 
with previous studies. For instance, Chang et al. demonstrated that WBVT can improve TUG times in older people with sarcopenia [87]. But in addition to TUG times, the effects of WBVT on GS and CS times were without significant effects in our study. It indicated that TUG maybe more sensitive to exercise, and the change of TUG times is more significant than that of other physical performance indicators such as GS and CS times at the same time. However, previous studies were inconsistent with our study on the result of the effects of WBVT on GS and CS times. Wei et al. demonstrated that 12-week WBV exercise not only can make a positive improvement on TUG times, but also GS and CS times [51]. In terms of the intervention period, we found that the total vibration intervention period in our included WBVT studies averaged 10 weeks, and 12 weeks in Wei et al.s study. Therefore, we speculated that the effect of WBVT on physical performance will be affected by time, and a suitable-long intervention period can comprehensively improve physical performance.

The fact of our meta-analysis failed to show a significant effect of WBVT on KES, but previous studies have opposite results for the effect of WBVT on KES in older people. For instance, a meta-analysis of 6 controlled clinical trials and RCTs by Wu et al. [88] and a meta-analysis of 12 studies ( 7 studies in younger people, mean ages of $11.8-37.7 \mathrm{yr}$; and 5 studies with older people, mean ages 60.7-77.6yr.) by Osawa et al. [89] both demonstrated that WBVT can lead to a significant improvement in lower body muscle strength as measured by KES in older people without and with sarcopenia [88, 89]. For such different results, it is speculated that different WBVT protocols may cause the differences of effects on KES. Firstly, the vibration frequency for the WBVT may have limited its ability to improve KES. In a study of eighty community-dwelling older adults with age-related muscle loss, Wei et al. compared different combinations of vibration frequencies $(20 \mathrm{~Hz}, 40 \mathrm{~Hz}, 60 \mathrm{~Hz})$ on knee extension performance. The results showed that the vibration frequency of $40 \mathrm{~Hz}$ was optimal in improving isokinetic knee extension performance [65]. However, in one third of the cited studies, the frequency of WBV was lower than $20 \mathrm{~Hz}$, which is likely too low to improve muscle strength. Thus, the lower frequency of WBVT among the studies reviewed in this systematic review and metaanalysis is a possible reason for the lack of significance in showing WBVT effect on KES. Secondly, the amplitude of WBV may also be a reason why WBVT has a non-significant effect on KES. In a meta-analysis of WBVT on muscle strength, Marin et al. observed that studies using lower amplitudes $(2-6 \mathrm{~mm})$ showed less efficacy on muscle strength compared with studies using higher amplitudes $(8-10 \mathrm{~mm})$ [90]. There have five of the six studies reviewed used amplitudes less than $6 \mathrm{~mm}(3-5 \mathrm{~mm})$, we speculated that the peak-to-peak displacement amplitude of the WBV was a factor for the lack of improvement on muscle strength. Thirdly, the exposure time of WBV can also be a reason why it cannot improve KES. A study by Da Silva-Grigoletto found that repeated 60-s bouts of WBVT with a total exposure time of $10 \mathrm{~min}$ was optimal than longer bout durations (i.e., $90 \mathrm{~s}$ ) with a total exposure time greater than $10 \mathrm{~min}$ on improving muscle function [91]. Accordingly, it may be considered that muscle fatigue will occur when the exposure time is too excessive which could have limited the efficacy of WBVT on KES [92]. However, in the studies cited in our study, the exposure time of WBV varied from 60- to 180 -s sets with a total exposure time of 5-15 min, and the different durations of WBV time ranging from 15 to $40 \mathrm{~min}$. The above three point of views are why we speculate that WBVT cannot significantly improve KES, and more RCTs should be conducted to explore the effects of WBVT on muscle strength in older people with sarcopenia. Thus, clinical evidence will be added to sufficiently illustrate the effect of WBVT on sarcopenia.

The results of our meta-analysis showed that MT was effective on improving lower limb muscle strength as measured by KES, TUG times and GS in older people with sarcopenia, but not CS times. A systematic review and meta-analysis study showed improvements on TUG times from MT (combined with resistance and balance training) in community-dwelling frail older people [83], meanwhile several clinical studies also showed that the effect of MT improved significantly on TUG times [33, 93] which is consistent with the result of our study. However, there still have some studies showed that MT cannot make a significant improvement on TUG times. For instance, Wang et al. showed that MT program did not significantly improve TUG compared with usual care in aged 80 years or over older people with sarcopenia [32]. For the result of GS, it is speculated that the training movements is an extremely relevant factor, which makes MT have a significant impact on GS. In terms of training movements, MT have more abundant and complex movements. Pojskic et al. demonstrated that complex training protocols that require multi joint movements and whole body transitions over a short distance were effective in improving response time in agility-based activities of young trained athletes [94]. It is plausible subjects who performed complex motor skills during MT had faster reaction times and had the agility to navigate complex environmental conditions (e.g., stepping over objects, carrying items while walking) needed to increase GS following exercise training [95]. Complex exercise training increases vestibular-driven signals needed for postural changes and the ability to maintain 
balance during postural changes is an important ability for GS [96]. No significant changes in the CS times were observed following MT in older people with sarcopenia in our study. As the movement of CS reflects the comprehensive ability of lower limbs to raise and lower the body from a seated position [97], such movements may not always be integrated into exercise training protocols. An example of appropriate exercise movements in MT are the squat which requires strength of the quadriceps, gluteus maximus, and hamstrings muscles, and the bench press which builds core strength. Several studies reviewed in this systematic review and meta-analysis included squats in MT studies [16, 33, 49, 68] protocols showed that the dose of exercise to improve CS performance was insufficient for older people with sarcopenia.

In our study, RT and MT both have significant effects on KES, TUG times and GS, and this result is consistent with previous studies [32, 49, 72, 80, 81, 83, 98]. According to the forest plot of the subgroup analyses in RT and MT with KES, TUG and GS as outcomes, we found that RT maybe have a more effective influence than MT due to RT's higher effect size than MT in each subgroup with KES, TUG, GS and CS. However, this is inconsistent with previous studies which showed that the effects of MT on muscle strength and physical performance were better than RT in healthy young man [73], or even MT have non-additive effect on muscle strength compared to the RT in athletes [74]. But so far, there have no studies exploring the comparison of RT and MT on muscle strength and physical performance in older people with sarcopenia. Thus, further research should conduct the comparison of these two modes exercises on muscle strength and physical performance in older people with sarcopenia.

In our study, WBVT only made a significant improvement on physical performance (TUG), but not effectively improved muscle strength. The above result we speculated that compared with MT, RT can improve muscle strength (KES) and physical performance (TUG times and GS) in older people with sarcopenia. Combined with above two speculations, the effect of WBVT on sarcopenia maybe not as good as RT and MT. To date, there have no studies to compare the effects of WBVT to RT or MT on sarcopenia, so according to the current findings, WBVT maybe not an effective alternative method for improving muscle strength and physical performance in older people with sarcopenia. This is mainly because there are few studies on the effect of WBVT on sarcopenia at present, so our study cannot include sufficient WBV studies that it is not enough to explain the effect of WBVT on sarcopenia. Therefore, further studies should be conducted to explore the effects of WBVT sarcopenia in older people.

\section{Limitations}

This study had some limitations. First, we did not assess the effects of the different exercise training modes on muscle mass due to differences in criteria, indicators and assessment methods used to determine muscle mass. For example, studies using AWGS or EWGSOP criteria to measure skeletal muscle mass (SSM) or appendicular skeletal muscle mass (ASM) used different measurement methods to determine muscle mass (e.g., bioelectrical impedance analysis (BIA) or dual-energy X-ray absorptiometry [DXA]). These different measurement methods make it difficult to compare muscle mass between studies. Future studies in this field should unify the criteria, indicators and assessment methods used to measure muscle mass to allow between study comparisons. Second, the high heterogeneity in some results of this study (e.g., GS $\left[\mathrm{I}^{2}=88 \%\right]$ and CS times $\left[\mathrm{I}^{2}=88 \%\right]$ ) might have been caused by differences in the assessment and exercise training programs. Researchers should conduct experiments in accordance with the standards and guidelines for assessing the studies independent and dependent variables to reduce the heterogeneity between studies and make them comparable. Third, we only included three exercise training modes (RT, MT, and WBVT) to explore the effects of different exercise training modes on muscle strength and physical performance outcomes in older people with sarcopenia. Other exercise training modes may prove beneficial in increasing muscle strength and physical performance. Moreover, differences in age, gender and/or factors related to aging may have moderated the effects of the three exercise training modes on the study outcomes in older people with sarcopenia. More studies should be conducted to explore the effects of additional exercise training modes and stratify the results by gender, age groups and gender-specific age groups to understand the effects of exercise training on muscle strength and physical performance in older people with sarcopenia.

\section{Conclusions}

In older people with sarcopenia, the findings show that resistance training (RT) and mixed training (MT) exercise training modes have positive effects on knee extension strength (KES) and physical performance tests of the Timed Up and Go (TUG) and gait speed (GS), but did not improve performance in the Chair Stand (CS). Whole Body Vibration training (WBVT) had a positive effect on the TUG times, but had no effects on KES, GS and CS outcomes. Plausible reasons can explain these findings to include differences in the exercise training movements, exercise-specific demands on the body, and variations in exercise training protocols. Overall, RT, MT and WBVT are worthwhile exercise modes to achieve 
various improvements on muscle strength and physical performance in older adults with sarcopenia. RT alone or with MT combined with other exercise training modes such as aerobic, balance and gait training may be better than WBVT to improve overall physical function in older people, especially those who are frail. Older people with age-related disabilities (e.g., dementia, osteoarthritis, and hemiplegia) and who may have difficulty performing RT and MT exercise training modes may benefit from WBVT in standing or sitting postures to maintain and/or improve aspects of physical function. These findings should be confirmed by high-quality randomized controlled trials (RCTs) that explore the effects of different exercise trainings modes and protocols on muscle strength and physical performance.

\section{Abbreviations}

AWGS: Asian Working Group for Sarcopenia; EWGSOP: European Working Group on Sarcopenia in Older People; PEDro: Physiotherapy Evidence Database; PRISMA: Preferred Reporting Items for Systematic Review and Meta-Analysis; PROSPERO: International Prospective Register of Systematic Reviews site; GRADE: Grades of Recommendation, Assessment, Development and Evaluation; CNKI: China National Knowledge Infrastructure; NHANES III: Third National Health and Nutrition Examination Survey; CDC: Centers for Disease Control and Prevention; BIA: Bioelectrical impedance analysis; DXA: Dual energy X-ray absorptiometry; RT: Resistance training; VT: Vibration training:WBV: Whole body vibration training: WBVT: Whole body vibration training; MT: Mixed training; AT: Aerobic training; ET: Endurance training; ETG: Exercise training group; CG: Control group; RCT: Randomized controlled trial; RCTs: Randomized controlled trials; LG: Low frequency and long exercise time group; MG: Medium frequency and medium exercise time group; HG: High frequency and short exercise time group; ASM: Appendicular skeletal muscle mass; ASM/ht': Appendicular skeletal muscle mass/height ${ }^{2}$; ALMI: Appendicular lean muscle mass index (ALMI = appendicular lean muscle mass/ height $\left.{ }^{2}\left[\mathrm{ALM} / \mathrm{m}^{2}\right]\right)$; AMI: Appendicular muscle mass index (AMI = appendicular muscle mass/height ${ }^{2}\left[\mathrm{AMM} / \mathrm{m}^{2}\right]$ ]); AMM: Appendicular muscle mass; $\mathrm{ASM}^{*}$ : $=0.2487$ (weight) +0.0483 (height) 0.1584 (hip circumference) +0.0732 (grip strength) + 2.5843(sex) + 5.8828; ASMl: Appendicular skeletal muscle mass index (ASMI = appendicular skeletal muscle mass $/$ height $^{2}\left[\mathrm{ASM} / \mathrm{m}^{2}\right]$ ); ASMI2 : $=$ appendicular skeletal muscle mass $/$ weight ${ }^{2 *} 100 \%\left[\mathrm{ASM} / \mathrm{kg}^{2}\right] ; \mathrm{ASMI} 4$ : $=[0.244$ (weight) +7.8 (height) +6.6 (sex)-0.098(age) + race-3.3]/body mass index (ASM/BMI); BMI: Body mass index; MM: Muscle mass; MMI: Muscle mass index (MMI $=$ muscle mass/height ${ }^{2}\left[\mathrm{MMI} / \mathrm{m}^{2}\right]$ ); SMI: Skeletal muscle mass index (SMI = skeletal muscle mass/ height2 [SMI/m2]); SMM: Skeletal muscle mass; CS: Chair Stand; GS: Gait speed; HGS: Handgrip strength; KES: Knee extension strength; TUG: Timed Up and Go; 4MWT: 4-m walk test; 5-STS: 5-chair sit to stand test; 6MWT: 6-m walk test; SPPB: Short Physical Performance Battery; ADL: Activities of daily living; UL: Upper limb; LL: Lower limb; M: Male; F: Female; W: Week; reps: Repetitions; RM: Repetition maximum; 1RM: One repetition maximum; maxHR: Maximum Heart Rate; RPE: Rated perceived exertion; FNT: Maximum theoretical force; SD: Standard deviations; SMDs: Standardized mean differences; WMD: Weighted mean differences; 95\% Cl: $95 \%$ confidence intervals.

\section{Supplementary Information}

The online version contains supplementary material available at https://doi. org/10.1186/s12877-021-02642-8.

Additional file 1. (DOCX 24.6 kb)

\section{Acknowledgements}

Not applicable.

\section{Authors' contributions}

LL, LM and YF participated in protocol design, data extraction, quality assessment, statistical analyses and manuscript preparation. AB participated in manuscript revision. NC and YL participated in protocol design, quality assessment, and manuscript revision. All authors have read and approved the final manuscript.

\section{Funding}

This meta-analysis is funded by special health research project of Shanghai Municipal Health Commission on the Health of Ageing, Woman and Children, "Exploration on the Screening and Rehabilitation Intervention Model for Sarcopenia among Community-dwelling Older People in Chongming District under the Medical Union Model" (No. 2020YJZX0137) and the project of Science and Technology Committee of Chongming District,"Effect of Exercise Intervention on Breast Cancer Patients in Chongming District (No. CKY2021-05).

\section{Availability of data and materials}

The datasets used and/or analysed during the current study are available from the corresponding author on reasonable request.

\section{Declarations}

Ethics approval and consent to participate

Not applicable (because this is a system review article).

Consent for publication

Not applicable.

\section{Competing interests}

The authors declare that they have no competing interests.

\section{Author details}

${ }^{1}$ Key Laboratory of Exercise and Health Sciences of Ministry of Education, Shanghai University of Sport, Shanghai 200438, China. ${ }^{2}$ Department of Rehabilitation, Xinhua Hospital Chongming Branch, Shanghai 202150, China.

${ }^{3}$ Department of Rehabilitation, Xinhua Hospital Affiliated to Shanghai Jiaotong University School of Medicine, Shanghai, China. ${ }^{4}$ College of Health Solutions, Arizona State University, Phoenix, AZ, USA.

Received: 27 August 2021 Accepted: 16 November 2021 Published online: 15 December 2021

\section{References}

1. Chen LK, Woo J, Assantachai P, Auyeung TW, Chou MY, lijima K, et al. Asian Working Group for Sarcopenia: 2019 consensus update on sarcopenia diagnosis and treatment. J Am Med Dir Assoc. 2020;21(3):300-7.

2. Papadopoulou SK, Tsintavis P, Potsaki P, Papandreou D. Differences in the prevalence of sarcopenia in community-dwelling, nursing home and hospitalized individuals. A systematic review and meta-analysis. J Nutr Health Aging. 2020;24(1):83-90.

3. Yeung SSY, Reijnierse EM, Pham VK, Trappenburg MC, Lim WK, Meskers CGM, et al. Sarcopenia and its association with falls and fractures in older adults: a systematic review and meta-analysis. J Cachexia Sarcopenia Muscle. 2019;10(3):485-500.

4. Harris R, Chang Y, Beavers K, Laddu-Patel D, Bea J, Johnson K, et al. Risk of fracture in women with sarcopenia, low bone mass, or both. J Am Geriatr Soc. 2017;65(12):2673-8.

5. Ohara DG, Pegorari MS, Santos NLOD, Silva CFR, Oliveira MSR, Matos AP, et al. Cross-sectional study on the association between pulmonary function and sarcopenia in Brazilian community-dwelling elderly from the Amazon region. J Nutr Health Aging. 2020;24(2):181-7.

6. Ida S, Kaneko R, Nagata H, Noguchi Y, Araki Y, Nakai M, et al. Association between sarcopenia and sleep disorder in older patients with diabetes. Geriatr Gerontol Int. 2019;19(5):399-403.

7. Peng TC, Chen WL, Wu LW, Chang YW, Kao TW. Sarcopenia and cognitive impairment: a systematic review and meta-analysis. Clin Nutr. 2020;39(9):2695-701. 
8. Rizzoli R, Reginster J-Y, Arnal J-F, Bautmans I, Beaudart C, BischoffFerrari $\mathrm{H}$, et al. Quality of life in sarcopenia and frailty. Calcif Tissue Int. 2013;93(2):101-20.

9. Kitamura A, Seino S, Abe T, Nofuji Y, Yokoyama Y, Amano H, et al. Sarcopenia: prevalence, associated factors, and the risk of mortality and disability in Japanese older adults. J Cachexia Sarcopenia Muscle. 2021;12(1):30-8.

10. Zhang XM, Zhang WW, Wang CH, Tao WY, Dou QL, Yang YZ. Sarcopenia as a predictor of hospitalization among older people: a systematic review and meta-analysis. BMC Geriatr. 2018;1 8(1):188.

11. Yang M, Liu Y, Zuo Y, Tang HR. Sarcopenia for predicting falls and hospitalization in community-dwelling older adults: EWGSOP versus EWGSOP2. Sci Rep. 2019;9(1):17636.

12. Gani F, Buettner S, Margonis GA, Sasaki K, Wagner D, Kim Y, et al. Sarcopenia predicts costs among patients undergoing major abdominal operations. Surgery. 2016;160(5):1162-71.

13. Janssen I, Shepard DS, Katzmarzyk PT, Roubenoff R. The healthcare costs of sarcopenia in the United States. J Am Geriatr Soc. 2004;52(3):80-5.

14. Cruz-Jentoft AJ, Sayer AA. Sarcopenia. Lancet. 2019;393(10191):2636-46.

15. Dent E, Morley JE, Cruz-Jentoft AJ, Arai H, Kritchevsky SB, Guralnik J, et al. International clinical practice guidelines for sarcopenia (ICFSR): screening, diagnosis and management. J Nutr Health Aging. 2018;22(10):1148-61.

16. Maruya K, Asakawa Y, Ishibashi H, Fujita H, Arai T, Yamaguchi H. Effect of a simple and adherent home exercise program on the physical function of community dwelling adults sixty years of age and older with pre-sarcopenia or sarcopenia. J Phys Ther Sci. 2016;28(11):3183-8.

17. Vlietstra L, Hendrickx W, Waters DL. Exercise interventions in healthy older adults with sarcopenia: a systematic review and meta-analysis. Australas J Ageing. 2018;37(3):169-83.

18. Bao WX, Sun Y, Zhang TF, Zou LL, Wu XH, Wang DM, et al. Exercise programs for muscle mass, muscle strength and physical performance in older adults with sarcopenia: a systematic review and meta-analysis. Aging Dis. 2020;11(4):863-73.

19. Nelson ME, Rejeski WJ, Blair SN, Duncan PW, Judge JO, King AC, et al. Physical activity and public health in older adults: recommendation from the American College of Sports Medicine and the American Heart Association. Med Sci Sports Exerc. 2007;39(8):1435-45.

20. World Health Organization. The global action plan on physical activity 2018-2030: more active people for a healthier world. World Health Organization; 2018. [Available from: https://apo.org.au/node/175331].

21. Vezzoli A, Mrakic-Sposta S, Montorsi M, Porcelli S, Vago P, Cereda F, et al. Moderate intensity resistive training reduces oxidative stress and improves muscle mass and function in older individuals. Antioxidants (Basel). 2019;8(10):431.

22. Chen HT, Wu HJ, Chen YJ, Ho SY, Chung YC. Effects of 8-week kettlebell training on body composition, muscle strength, pulmonary function, and chronic low-grade inflammation in elderly women with sarcopenia. Exp Gerontol. 2018;112:112-8.

23. Tsekoura M, Billis E, Tsepis E, Dimitriadis Z, Matzaroglou C, Tyllianakis M, et al. The effects of group and home-based exercise programs in elderly with sarcopenia: a randomized controlled trial. J Clin Med. 2018;7(12):480.

24. Beckwée D, Delaere A, Aelbrecht S, Baert V, Beaudart C, Bruyere O, et al. Exercise interventions for the prevention and treatment of sarcopenia. A systematic umbrella review. J Nutr Health Aging. 2019;23(6):494-502.

25. Mello RGB, Corte RRD, Gioscia J, Moriguchi EH. Effects of physical exercise programs on sarcopenia management, dynapenia, and physical performance in the elderly: 5 systematic review of randomized clinical trials. J Aging Res. 2019;2019:1959486.

26. Miyazaki R, Takeshima T, Kotani K. Exercise intervention for antisarcopenia in community-dwelling older people. J Clin Med Res. 2016;8(12):848-53.

27. Lai CC, Tu YK, Wang TG, Huang YT, Chien KL. Effects of resistance training, endurance training and whole-body vibration on lean body mass, muscle strength and physical performance in older people: a systematic review and network meta-analysis. Age Ageing. 2018;47(3):367-73.

28. Šarabon N, Kozinc Ž, Löfler S, Hofer C. Resistance exercise, electrical muscle stimulation, and whole-body vibration in older adults: systematic review and meta-analysis of randomized controlled trials. J Clin Med. 2020:9(9):2902.
29. Yang L, Yao XM, Shen J, Sun GF, Sun Q, Tian XL, et al. Comparison of revised EWGSOP criteria and four other diagnostic criteria of sarcopenia in Chinese community-dwelling elderly residents. Exp Gerontol. 2020;130:110798.

30. Nawi SNM, Khow KS, Lim WS, Yu SC. Screening tools for sarcopenia in community-dwellers: a scoping review. Ann Acad Med Singap. 2019:48(7):201-16.

31. Reiss J, Iglseder B, Kreutzer M, Weilbuchner I, Treschnitzer W, Kässmann H, et al. Case finding for sarcopenia in geriatric inpatients: performance of bioimpedance analysis in comparison to dual X-ray absorptiometry. BMC Geriatr. 2016;16:52.

32. Wang R, Liang Y, Jiang J, Chen M, Li L, Yang H, et al. Effectiveness of a short-term mixed exercise program for treating sarcopenia in hospitalized patients aged 80 years and older: a prospective clinical trial. J Nutr Health Aging. 2020;24(10):1087-93.

33. Makizako H, Nakai Y, Tomioka K, Taniguchi Y, Sato N, Wada A, et al. Effects of a multicomponent exercise program in physical function and muscle mass in sarcopenic/pre-sarcopenic adults. J Clin Med. 2020;9(5):1386.

34. Hanson ED, Srivatsan SR, Agrawal S, Menon KS, Delmonico MJ, Wang MQ, et al. Effects of strength training on physical function: influence of power, strength, and body composition. J Strength Cond Res. 2009;23(9):262737 Available from: https://www.cochranelibrary.com/central/doi/10. 1002/central/CN-00728802/full.

35. Raya SA, Raya AA, Kareem AA. Effects of physical activity on inflammation skeletal muscle strength/function (sarcopenia) and fat infiltration (sarcopenic obesity) in older adults with knee osteoarthritis: a randomized controlled trial. Ann Rheum Dis. 2013;72:690.

36. Genest F, Lindström S, Luksche N, Jakob F, Seefried L. Combined efficacy of different exercise interventions in osteosarcopenic men. J Bone Mineral Res. 2017:32:S398.

37. Cho JK, Choi YJ, Sajgalik P, No MH, Lee SH, Kim SJ, et al. Exercise as a therapeutic strategy for sarcopenia in heart failure: insights into underlying mechanisms. Cells. 2020;9(10):2284.

38. Vrabel M. Preferred reporting items for systematic reviews and metaanalyses. Oncol Nurs Forum. 2015;42(5):552-4.

39. Maher CG, Sherrington C, Herbert RD, Moseley AM, Elkins M. Reliability of the PEDro scale for rating quality of randomized controlled trials. Phys Ther. 2003;83(8):713-21.

40. Ahmed I, Sutton AJ, Riley RD. Assessment of publication bias, selection bias, and unavailable data in meta-analyses using individual participant data: a database survey. BMJ (Clin Res ed). 2012;344:d7762.

41. Felsom DT. Bias in meta-analytic research. J Clin Epidemiol. 1992;45(8):885-92.

42. GRADE handbook for grading quality of evidence and strength of recommendations: The GRADE Working Group 2013. 2013. [Available from: https://gdt.gradepro.org/app/handbook/handbook.html].

43. Guyatt G, Oxman AD, Akl EA, Kunz R, Vist G, Brozek J, et al. GRADE guidelines: 1. Introduction-GRADE evidence profiles and summary of findings tables. J Clin Epidemiol. 2011;64(4):383-94.

44. Guyatt GH, Oxman AD, Vist G, Kunz R, Brozek J, Alonso-Coello P, et al. GRADE guidelines: 4. Rating the quality of evidence--study limitations (risk of bias). J Clin Epidemiol. 2011:64(4):407-15.

45. Guyatt GH, Oxman AD, Kunz R, Woodcock J, Brozek J, Helfand M, et al. GRADE guidelines: 7. Rating the quality of evidence--inconsistency. J Clin Epidemiol. 2011;64(12):1294-302.

46. Guyatt GH, Oxman AD, Kunz R, Woodcock J, Brozek J, Helfand M, et al. GRADE guidelines: 8 . Rating the quality of evidence--indirectness. J Clin Epidemiol. 2011;64(12):1303-10.

47. Guyatt GH, Oxman AD, Kunz R, Brozek J, Alonso-Coello P, Rind D, et al. GRADE guidelines 6. Rating the quality of evidence--imprecision. J Clin Epidemiol. 2011;64(12):1283-93.

48. Guyatt GH, Oxman AD, Montori V, Vist G, Kunz R, Brozek J, et al. GRADE guidelines: 5 . Rating the quality of evidence--publication bias. J Clin Epidemiol. 2011;64(12):1277-82.

49. Chen HT, Chung YC, Chen YJ, Ho SY, Wu HJ. Effects of different types of exercise on body composition, muscle strength, and IGF-1 in the elderly with sarcopenic obesity. J Am Geriatr Soc. 2017;65(4):827-32.

50. Bellomo RG, lodice P, Maffulli N, et al. Muscle strength and balance training in sarcopenic elderly: a pilot study with randomized controlled trial. Eur. J Inflamm. 2013;11(1):193-201. 
51. Wei N, Pang MY, Ng SS, Ng GY. Optimal frequency/time combination of whole body vibration training for developing physical performance of people with sarcopenia: a randomized controlled trial. Clin Rehabil. 2017;31(10):1313-21.

52. Zhu LY, Chan R, KwokT, Cheng KCC, Ha A, Woo J. Effects of exercise and nutrition supplementation in community-dwelling older Chinese people with sarcopenia: a randomized controlled trial. Age Ageing. 2019;48(2):220-8.

53. Zhu YQ. Tai chi and whole-body vibration therapy on elderly: a clinical randomized controlled trial. China National Knowledge Infrastructure; 2016.

54. Iranzo MȦCl, Balasch-Bernat M, Tortosa-Chuliá MÁ, Balasch-Parisi S. Effects of resistance training of peripheral muscles versus respiratory muscles in older adults with sarcopenia who are institutionalized: a randomized controlled trial. J Aging Phys Act. 2018;26(4):637-46.

55. Shao WH, Gao LX, Wang SX, Lyu CX, Yao LX, Li SB. Effects of vitamin D combined with resistance training on skeletal muscle mass, activities of daily living and serological indices in elderly patients with sarcopenia. Chin J Multiple Organ Dis Elderly. 2020;19(09):656-60.

56. Vikberg S, Sörlén N, Brandén L, Johansson J, Nordström A, Hult A, et al. Effects of resistance training on functional strength and muscle mass in 70-year-old individuals with pre-sarcopenia: a randomized controlled trial. J Am Med Dir Assoc. 2019;20(1):28-34.

57. Liao CD, Tsauo JY, Huang SW, Ku JW, Hsiao DJ, Liou TH. Effects of elastic band exercise on lean mass and physical capacity in older women with sarcopenic obesity: a randomized controlled trial. Sci Rep. 2018;8(1):2317.

58. Bagheri R, Moghadam BH, Church DD, Tinsley GM, Eskandari M, Moghadam BH, et al. The effects of concurrent training order on body composition and serum concentrations of follistatin, myostatin and GDF11 in sarcopenic elderly men. Exp Gerontol. 2020;133:110869.

59. Zhu YQ, Peng N, Zhou M, Liu PP, Qi XL, Wang N, et al. Tai chi and wholebody vibrating therapy in sarcopenic men in advanced old age: a clinical randomized controlled trial. Eur J Ageing. 2019;16(3):273-82.

60. Moghadam BH, Bagheri R, Ashtary-Larky D, Tinsley GM, Eskandari M, Wong $A$, et al. The effects of concurrent training order on satellite cell-related markers, body composition, muscular and cardiorespiratory fitness in older men with sarcopenia. J Nutr Health Aging. 2020;24(7):796-804.

61. Wei N, Ng S, Ng G, Lee R, Lau M, Pang M. Whole-body vibration training improves muscle and physical performance in community dwelling with sarcopenia: a randomized controlled trial. Int J Phys Ther Rehabil. 2016;2(1):116.

62. Hamaguchi K, Kurihara T, Fujimoto M, lemitsu M, Sato K, Hamaoka T, et al. The effects of low-repetition and light-load power training on bone mineral density in postmenopausal women with sarcopenia: a pilot study. BMC Geriatr. 2017;17(1):102.

63. Kim H, Suzuki T, Saito K, Yoshida H, Kojima N, Kim M, et al. Effects of exercise and tea catechins on muscle mass, strength and walking ability in community-dwelling elderly Japanese sarcopenic women: a randomized controlled trial. Geriatr Gerontol Int. 2013;13(2):458-65.

64. Liao CD, Tsauo JY, Lin LF, Huang SW, Ku JW, Chou LC, et al. Effects of elastic resistance exercise on body composition and physical capacity in older women with sarcopenic obesity: a CONSORT-compliant prospective randomized controlled trial. Medicine (Baltimore). 2017:96(23):e7115.

65. Wei N, Pang MY, Ng SS, Ng GY. Optimal frequency/time combination of whole-body vibration training for improving muscle size and strength of people with age-related muscle loss (sarcopenia): a randomized controlled trial. Geriatr Gerontol Int. 2016;17(10):1412-20.

66. Kim HK, Suzuki T, Saito K, Yoshida H, Kobayashi H, Kato H, et al. Effects of exercise and amino acid supplementation on body composition and physical function in community-dwelling elderly Japanese sarcopenic women: a randomized controlled trial. J Am Geriatr Soc. 2012;60(1):16-23.

67. Kim H, Kim M, Kojima N, Fujino K, Hosoi E, Kobayashi H, et al. Exercise and nutritional supplementation on community-dwelling elderly Japanese women with sarcopenic obesity: a randomized controlled trial. J Am Med Dir Assoc. 2016;17(11):1011-9.

68. Park J, Kwon Y, Park H. Effects of 24-week aerobic and resistance training on carotid artery intima-media thickness and flow velocity in elderly women with sarcopenic obesity. J Atheroscler Thromb. 2017;24(11):1117-24.

69. Vasconcelos KSS, Dias JMD, Araújo MC, Pinheiro AC, Moreira BS, Dias RC. Effects of a progressive resistance exercise program with high-speed component on the physical function of older women with sarcopenic obesity: a randomized controlled trial. Braz J Phys Ther. 2016;20(5):432-40.

70. Hassan BH, Hewitt J, Keogh JWL, Bermeo S, Duque G, Henwood TR. Impact of resistance training on sarcopenia in nursing care facilities: a pilot study. Geriatr Nurs. 2015;37(2):116-21.

71. Shahar S, Kamaruddin NS, Badrasawi M, Sakian NIM, Manaf ZA, Yassin Z, et al. Effectiveness of exercise and protein supplementation intervention on body composition, functional fitness, and oxidative stress among elderly Malays with sarcopenia. Clin Interv Aging. 2013;8:1365-75.

72. Qi M, Moyle W, Jones C, Weeks B. Tai chi combined with resistance training for adults aged 50 years and older: a systematic review. J Geriatr Phys Ther. 2020;43(1):32-41.

73. Rosenberger A, Beijer Å, BJ, Schoenau E, Mester J, Rittweger J, Zange J. Changes in muscle cross-sectional area, muscle force, and jump performance during 6 weeks of progressive whole-body vibration combined with progressive, high intensity resistance training. J Musculoskelet Neuronal Interact. 2017;17(2):38-49.

74. Arora NK, Sharma S, Saifi S, Sharma S, Arora IK. Effects of combined whole body vibration and resistance training on lower quadrants electromyographic activity, muscle strength and power in athletes. Foot (Edinb). 2021;49:101844.

75. Janssen I, Baumgartner RN, Ross R, Rosenberg $\|_{\text {, Roubenoff R. Skeletal }}$ muscle cutpoints associated with elevated physical disability risk in older men and women. Am J Epidemiol. 2004(4):413-21.

76. Chung JY, Kang HT, Lee DC, Lee HR, Lee YJ. Body composition and its association with cardiometabolic risk factors in the elderly: a focus on sarcopenic obesity. Arch Gerontol Geriatr. 2013;56(1):270-8.

77. Tyrovolas S, Koyanagi A, Olaya B, Ayuso-Mateos JL, Miret M, Chatterii S, et al. The role of muscle mass and body fat on disability among older adults: a cross-national analysis. Exp Gerontol. 2015;69:27-35.

78. Fried LP, Tangen CM, Walston J, Newman AB, Hirsch C, Gottdiener J, et al. Frailty in older adults: evidence for a phenotype. J Gerontol A Biol Sci Med Sci. 2001;56(3):M146-M56.

79. Sitjà-Rabert M, Rigau D, Vanmeerghaeghe AF, Romero-Rodríguez D, Subirana MB, Bonfill X. Efficacy of whole body vibration exercise in older people: a systematic review. Disabil Rehabil. 2012;34(11):883-93.

80. Yamada M, Kimura Y, Ishiyama D, Nishio N, Otobe Y, Tanaka T, et al. Synergistic effect of bodyweight resistance exercise and protein supplementation on skeletal muscle in sarcopenic or dynapenic older adults. Geriatr Gerontol Int. 2019;19(5):429-37.

81. Osuka Y, Fujita S, Kitano N, Kosaki K, Seol J, Sawano Y, et al. Effects of aerobic and resistance training combined with fortified milk on muscle mass, muscle strength, and physical performance in older adults: a randomized controlled trial. J Nutr Health Aging. 2017;21 (10):1349-57.

82. Dalgas U, Stenager E, Jakobsen J, Petersen T, Hansen HJ, Knudsen C, et al. Resistance training improves muscle strength and functional capacity in multiple sclerosis. Neurology. 2009;73(18):1478-84.

83. Zhang YX, Zhang YQ, Du SZ, Wang QL, Xia HZ, Sun R. Exercise interventions for improving physical function, daily living activities and quality of life in community-dwelling frail older adults: a systematic review and meta-analysis of randomized controlled trials. Geriatr Nurs. 2020;41(3):261-73.

84. Stoever K, Heber A, Eichberg S, Brixius K. Influences of resistance training on physical function in older, obese men and women with sarcopenia. J Geriatr Phys Ther. 2018:41(1):20-7.

85. Soligon SD, Silva DG, Bergamasco JGA, Angleri V, Júnior RAM, Dias NF, et al. Suspension training vs. traditional resistance training: effects on muscle mass, strength and functional performance in older adults. Eur J Appl Physiol. 2020;120(10):2223-32.

86. Vitale JA, Bonato M, Borghi S, Messina C, Albano D, Corbetta S, et al. Home-based resistance training for older subjects during the COVID-19 outbreak in Italy: preliminary results of a six-months RCT. Int J Environ Res Public Health. 2020;17(24).

87. Chang SF, Lin PC, Yang RS, Yang RJ. The preliminary effect of whole-body vibration intervention on improving the skeletal muscle mass index, physical fitness, and quality of life among older people with sarcopenia. BMC Geriatr. 2018;18(1):17.

88. Wu S, Ning HT, Xiao SM, Hu MY, Wu XY, Deng HW, et al. Effects of vibration therapy on muscle mass, muscle strength and physical function in older adults with sarcopenia: a systematic review and meta-analysis. Eur Rev Aging Phys Act. 2020;17:14 
89. Osawa Y, Oguma Y, Ishii N. The effects of whole-body vibration on muscle strength and power: a meta-analysis. J Musculoskelet Neuronal Interact. 2013;13(3):380-90.

90. Marín PJ, Rhea MR. Effects of vibration training on muscle strength: a meta-analysis. J Strength Cond Res. 2010;24(2):548-56.

91. Silva-Grigoletto MED, Hoyo MD, Sañudo B, Carrasco L, García-Manso JM. Determining the optimal whole-body vibration dose-response relationship for muscle performance. J Strength Cond Res. 2011;25(12):3326-33.

92. Rittweger J, Beller G, Felsenberg D. Acute physiological effects of exhaustive whole-body vibration exercise in man. Clin Physiol. 2000;20(2):134-42.

93. Lin SF, Sung HC, Li TL, Hsieh TC, Lan HC, Perng SJ, et al. The effects of tai-chi in conjunction with thera-band resistance exercise on functional fitness and muscle strength among community-based older people. J Clin Nurs. 2015;24(9-10):1357-66.

94. Pojskic H, Pagaduan J, Uzicanin E, Separovic V, Spasic M, Foretic N, et al. Reliability, validity and usefulness of a new response time test for agility-based sports: a simple vs. complex motor task. J Sports Sci Med. 2019;18(4):623-35.

95. Forte R, Vito GD, Boreham CAG. Reliability of walking speed in basic and complex conditions in healthy, older community-dwelling individuals. Aging Clin Exp Res. 2021;33(2):311-7.

96. Kennefick M, McNeil CJ, Burma JS, Copeland PV, PV D, Dalton BH. Modulation of vestibular-evoked responses prior to simple and complex arm movements. Exp Brain Res. 2020;238(4):869-81.

97. Pinheiro PA, Carneiro JAO, Coqueiro RS, Pereira R, Fernandes MH. "chair stand test" as simple tool for sarcopenia screening in elderly women. J Nutr Health Aging. 2016;20(1):56-9.

98. Del Campo M, Cervantes J, Habacuc Macías Cervantes M, Monroy TR. Effect of a resistance training program on sarcopenia and functionality of the older adults living in a nursing home. J Nutr Health Aging. 2019;23(9):829-36.

\section{Publisher's Note}

Springer Nature remains neutral with regard to jurisdictional claims in published maps and institutional affiliations.

Ready to submit your research? Choose BMC and benefit from:

- fast, convenient online submission

- thorough peer review by experienced researchers in your field

- rapid publication on acceptance

- support for research data, including large and complex data types

- gold Open Access which fosters wider collaboration and increased citations

- maximum visibility for your research: over $100 \mathrm{M}$ website views per year

At BMC, research is always in progress.

Learn more biomedcentral.com/submissions 